\title{
Magnetic Resonance Image Example-Based Contrast Synthesis
}

\author{
Snehashis Roy, Student Member, IEEE, Aaron Carass*, Member, IEEE, and Jerry L. Prince, Fellow, IEEE
}

\begin{abstract}
The performance of image analysis algorithms applied to magnetic resonance images is strongly influenced by the pulse sequences used to acquire the images. Algorithms are typically optimized for a targeted tissue contrast obtained from a particular implementation of a pulse sequence on a specific scanner. There are many practical situations, including multi-institution trials, rapid emergency scans, and scientific use of historical data, where the images are not acquired according to an optimal protocol or the desired tissue contrast is entirely missing. This paper introduces an image restoration technique that recovers images with both the desired tissue contrast and a normalized intensity profile. This is done using patches in the acquired images and an atlas containing patches of the acquired and desired tissue contrasts. The method is an example-based approach relying on sparse reconstruction from image patches. Its performance in demonstrated using several examples, including image intensity normalization, missing tissue contrast recovery, automatic segmentation, and multimodal registration. These examples demonstrate potential practical uses and also illustrate limitations of our approach.
\end{abstract}

Index Terms - Image restoration, magnetic resonance imaging (MRI), neuroimaging, sparse reconstruction.

\section{INTRODUCTION}

$\mathbf{M}$ AGNETIC resonance (MR) imaging (MRI) is widely used to image the brain. Postprocessing of MR brain images, e.g., image segmentation [1]-[6] and image registration [7]-[11], has been used for many scientific purposes such as furthering our understanding of normal aging [12], [13], disease progression [14], [15], population analysis [16], and prognosis [17]. It is well known that image analysis algorithms are routinely optimized for a particular type of scan (or collection of scans), which could include specification of the tissue contrast [e.g., T1-weighted (T1-w) versus T2-weighted (T2-w)], image resolution, pulse sequence parameters, field strength of the scanner, and even the particular scanner manufacturer. Although many algorithms claim to be robust to variations in the

Manuscript received August 06, 2013; accepted September 07, 2013. Date of publication September 16, 2013; date of current version November 25, 2013. This work is supported by the NIH/NIBIB under Grant 1R21EB012765. Asterisk indicates corresponding author.

S. Roy is with the Henry Jackson Foundation, Bethesda, MD 20817 USA

*A. Carass is with the Department of Electrical and Computer Engineering, Johns Hopkins University, Baltimore, MD 21218 USA (e-mail: aaron_carass@jhu.edu).

J. L. Prince is with the Department of Electrical and Computer Engineering, Johns Hopkins University, Baltimore, MD 21218 USA (e-mail: prince@jhu. edu).

Color versions of one or more of the figures in this paper are available online at http://ieeexplore.ieee.org.

Digital Object Identifier 10.1109/TMI.2013.2282126 input images, inevitably there will be performance degradation as the input images deviate from a perfect match to the images that were used to carry out the algorithm optimization.

There are many practical situations, including multi-institution trials [18]-[20], rapid emergency scans [21], and scientific use of historical data [12], where the images are not acquired according to an optimal protocol for a given image analysis algorithm. As well, a particular tissue contrast that is necessary for a given algorithm might have been omitted for a given study or for a particular patient acquisition. In such cases, investigators and clinicians have three choices. First, they might apply the algorithm on available images and accept a sub-optimal result. Second, they might remove patients with inadequate image data from the study. Third, they might design a new image analysis algorithm that will work equally well on the image data that is available to them. Upon consideration of these options, it seems that each choice leads to concerns, and an alternative is highly desirable. This paper is concerned with a fourth alternative: to recover an image with the desired tissue contrast and intensity profile using the available images and prior information. There are three general approaches described in the literature to carry out this fourth alternative, which we briefly describe next. The method we describe in this paper offers an approach with significant advantages over existing approaches including improved performance and greater applicability.

The most common approach to recovery of images with a desired tissue contrast and intensity profile is histogram matching [22]-[28]. Some manner of histogram matching is used as a preprocessing step in nearly every neuroimage segmentation and registration pipeline because they do improve the accuracy and consistency of results [30], [31]. However, its use becomes questionable when applied to data acquired with different pulse sequences, especially when pathology is present, and its use in volumetric analysis may lead to incorrect results simply because the histograms are forced to follow that of a target image which itself contains certain ratios of tissue types. One way to address this last concern is to first segment brain structures and match their individual histograms to a registered atlas [32]. Although this method produces consistent sub-cortical segmentations over data sets acquired under different scanners, it requires a detailed segmentation of the images before intensity normalization has been applied, which leads to a "chicken and egg" situation. It is clearly more desirable to normalize the image intensities prior to any significant image analysis algorithms have been applied.

A second approach to recover images with a desired tissue contrast and intensity profile is to acquire multiple images of the subject with predefined, precisely calibrated acquisition parameters [33]. Given this set of images, tissue proton density $P_{D}$ 

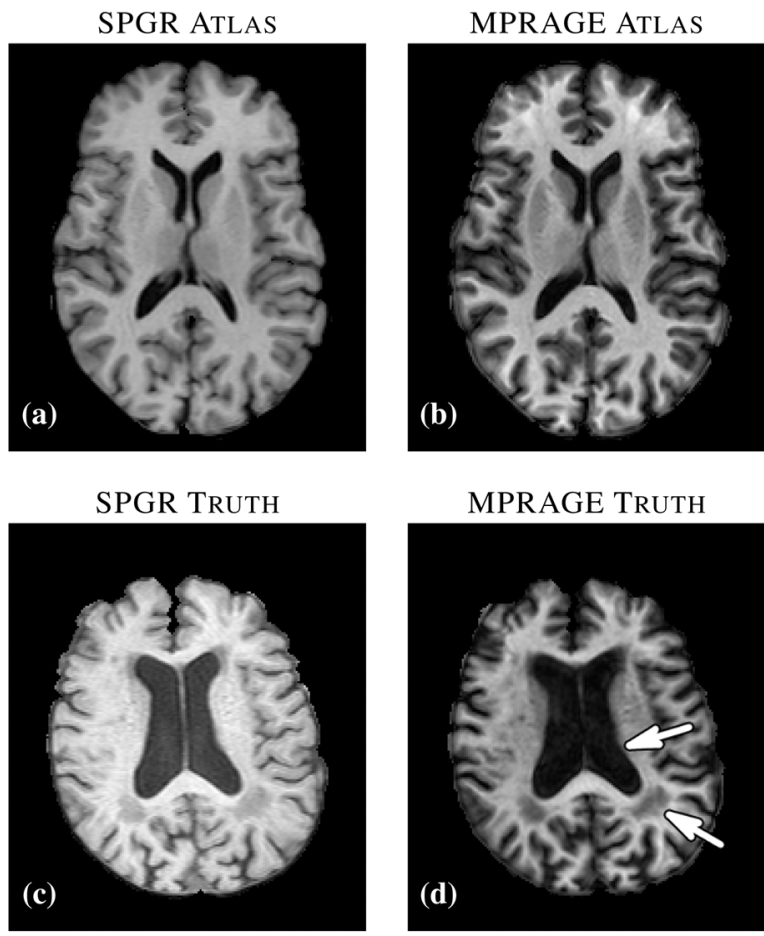

SYNTHETIC MPRAGE FROM REGISTRATION

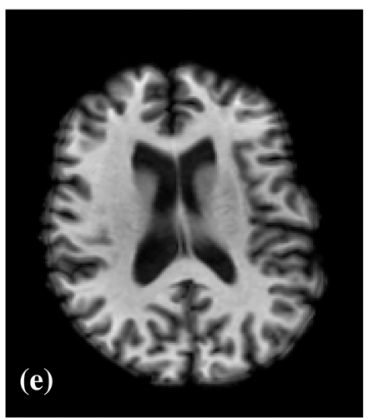

SYNTHETIC MPRAGE FROM MIMECS

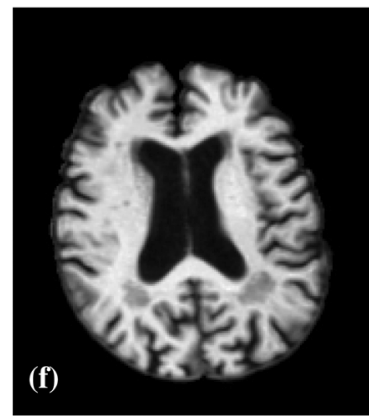

Fig. 1. (a) Atlas T1-weighted SPGR and (b) its corresponding T1-weighted MPRAGE. (c) Subject T1-weighted SPGR scan and (d) its T1-weighted MPRAGE image. Atlas SPGR is deformably registered (using SyN [7]) to the subject SPGR. This deformation is applied to the atlas MPRAGE to obtain (e) a synthetic subject MPRAGE. (f) Synthetic MPRAGE image generated by our algorithm, MIMECS.

and relaxation parameters $T_{1}, T_{2}$, and $T_{2}^{*}$ can be estimated by inverting the pulse sequence equations [34], and calibrated images with the desired tissue contrast can be computed by using these estimated parameters in the appropriate mathematical equation derived from the imaging physics [35]. This approach has three disadvantages. First, the imaging equations are typically only an approximation to what is used in practice, and therefore the acquired data may not be accurately related to the underlying tissue parameters by the assumed imaging equation. Second, the required voxel-wise mathematical inversion is generally poorly conditioned, which means that noise and artifacts can have a large impact on the accuracy of estimating the underlying tissue parameters. Third, in large imaging studies with many clinical scenarios it is difficult to acquire the source image data with consistent, predefined image acquisition parameters. Because of these problems, most image processing algorithms are not designed to exploit the existence of underlying tissue parameter estimates nor are most studies and clinical scans designed to exploit this approach to tissue contrast normalization.

Another way to recover a target tissue contrast and intensity profile is to register the subject to a multiple-contrast atlas and to transfer the desired contrast intensities from the atlas space to the subject space [36]. This approach enjoys great popularity not only for transferring tissue contrasts but also for transferring segmented tissue labels [37], and is tacitly assumed by many researchers to be the best current approach for generation of images with alternate tissue contrast. However, this approach does not produce an acceptable result if the registration result is poor or if there are anomalies or unknown tissues in the subject that are not present or spatially distributed differently in the atlas. In the example shown in Fig. 1, a T1-w spoiled gradient recalled (SPGR) atlas [Fig. 1(a)] was registered to a similar contrast subject image [Fig. 1(c)] using the (inverse consistent and diffeomorphic) SyN registration algorithm [7]. The atlas magnetization prepared rapid gradient echo (MPRAGE) contrast intensities [Fig. 1(b)] were then mapped through the transformation to the subject space, yielding a synthetic subject MPRAGE image [Fig. 1(e)]. By comparing this result to the subject's true MPRAGE image [Fig. 1(d)], it is apparent that this approach failed to adequately define the ventricles (Fig. 1(d), arrow adjacent to the ventricles) due to shortcomings in the registration, and it also failed to represent the WM lesions that are present in the original image (Fig. 1(d), arrow denoting the lesion posterior to the ventricles) because such lesions are not present in the atlas. The image produced using our approach [Fig. 1(f)] shows neither of these problems and compares quite favorably in overall contrast and appearance to the true MPRAGE.

The method proposed in this paper, $\underline{\mathbf{M}} \mathrm{R}$ image examplebased contrast synthesis (MIMECS), combines the idea of example-based image hallucination [38], [39] with nonlocal means [40]-[42] and sparse priors [43], [44] to synthesize MR contrasts using patches - i.e., small regions of the image- from a multiple-contrast atlas (also called a textbook in [36]). The core idea of MIMECS is that each subject patch can be matched to a combination of a few relevant patches from a dictionary in a nonlocal fashion [43], [45], [46]. It is different from classical histogram matching in the sense that a patch can be thought of as a feature vector of its center voxel, thus including local neighborhood information for that voxel. We show the results of seven case studies which demonstrate potential uses and limitations of MIMECS in realistic scientific and clinical scenarios.

There are several advantages of MIMECS compared to previous synthesis methods. First, it is a completely automatic preprocessing step that can precede any image processing task. Second, there is no need to use multiple, calibrated pulse sequences in order to estimate underlying tissue parameters. Third, even though MIMECS uses an atlas, there is no need to carry out subject-to-atlas registration, thus avoiding potential errors due to misregistration or missing tissues. Finally, it does not require any segmentation of the subject. This last point, detailed in Section III-C, is a unique feature of this work improving on our previous patch-based results [47]-[49], and 


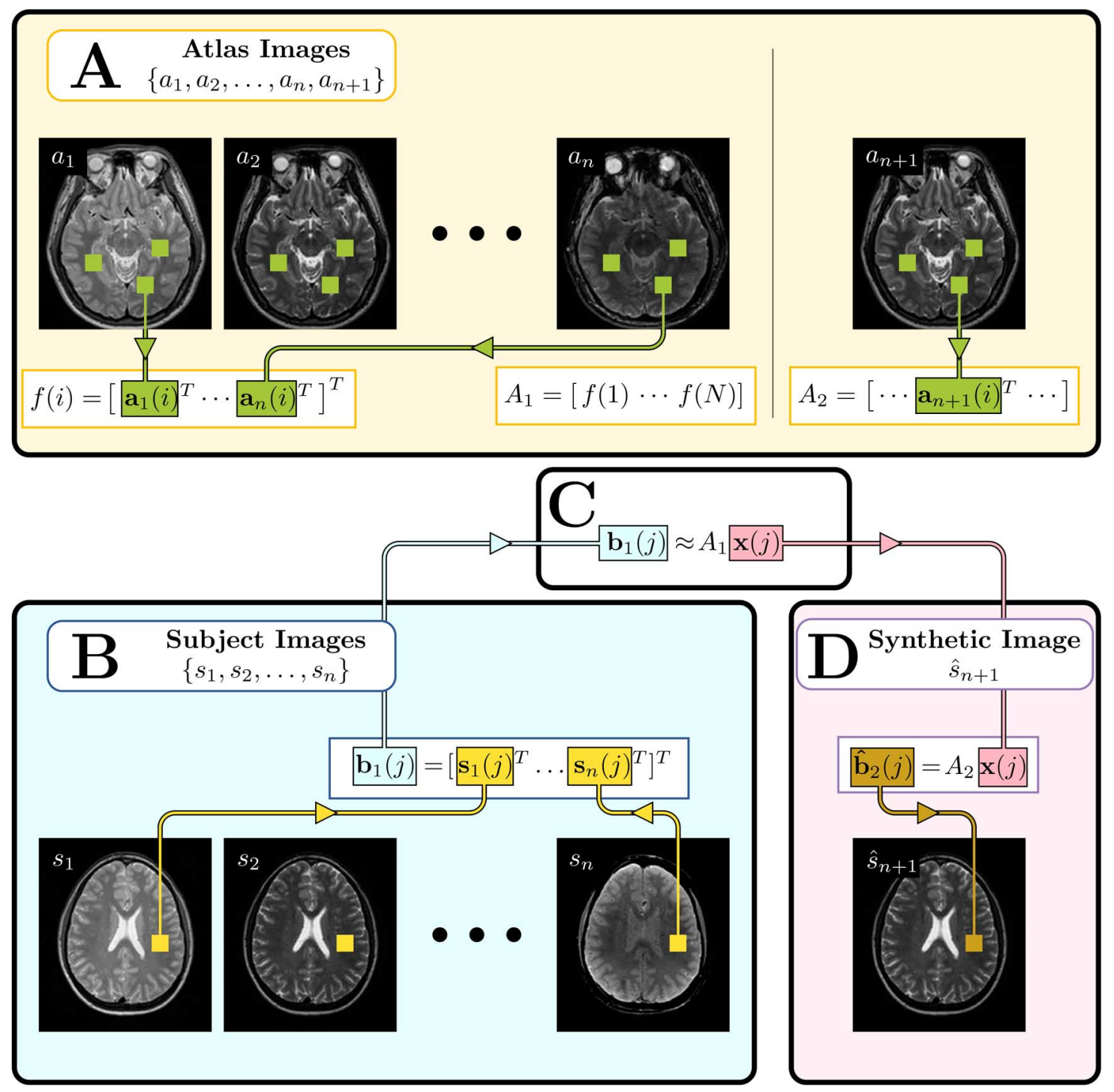

Fig. 2. Illustration of the MIMECS algorithm. Region of the image labeled A, shows the construction of the atlases $A_{1}$ and $A_{2}$, see Section III-A for details Region B shows the input subject images converted into patches $\mathbf{b}_{1}(j)$. Region C denotes the estimation of the coefficients $\mathbf{x}(j)$ which relate $\mathbf{b}_{1}(j)$ to the patches of the atlas $A_{1}$. Finally, region $\mathrm{D}$ shows the computation of the synthetic image $\hat{s}_{n+1}$ by using the learned coefficients $\mathbf{x}(j)$ to compute the patch $\hat{\mathbf{b}}_{2}(j)$ based on the atlas $A_{2}$. Section III-B describes regions $\mathrm{B}, \mathrm{C}$, and $\mathrm{D}$.

is important in the field of sparse reconstruction as it alleviates the need for atlas training.

The paper is organized as follows. First, we summarize the idea of sparse priors and establish some notation that will be used throughout the paper. Then the atlas-based patch matching synthesis algorithm is explained in a sparse priors paradigm, Fig. 2 provides a high level overview of the algorithm. Finally, we demonstrate the applicability of MIMECS on a variety of case studies.

\section{BACKGROUND}

Sparse signal reconstruction maintains that because most signals are sparse in some way it is not necessary to observe the full signal in order to accurately reconstruct it. The idea has been successfully applied to many image processing algorithms including denoising [46], image restoration [50], [51], and super- resolution [43], often improving on state-of-the-art algorithms. For example in [46], authors use a K-SVD algorithm [52] to generate an overcomplete set of bases from natural image patches and apply it to a denoising problem. Dictionary-based patch matching methods [53], [54] are also popular for natural image restoration. In our case, we build an overcomplete dictionary using patches from human brain images to both normalize a given contrast and synthesize alternate MR tissue contrasts. This section presents the mathematical formulation and key results that are needed to explain our approach.

Suppose we want to reconstruct a vector $\mathbf{x} \in \mathbb{R}^{N}$ that is $s$-sparse, i.e., having at most $s$ nonzero elements, and our observations are $\mathbf{b}=A \mathbf{x}$, where $\mathbf{b} \in \mathbb{R}^{d}, s<d<N$, and $A \in$ $\mathbb{R}^{d \times N}$. Since this is an under-determined system, we cannot directly invert the system to find $\mathbf{x}$, and additional penalties or constraints must therefore be used. Since $\mathbf{x}$ is known to be sparse, it makes sense to formulate an objective function that 
tries to find a sparse solution that is also consistent with the measurements, as follows:

$$
\hat{\mathbf{x}}=\min \|\mathbf{x}\|_{0} \text { such that }\|\mathbf{b}-A \mathbf{x}\|_{2}^{2}<\epsilon_{1} .
$$

Here, $\epsilon_{1}$ is the noise level in the measurements, $\|\cdot\|_{p}$ is the $\ell_{p}$ norm; in particular, $\|\mathbf{x}\|_{0}$ is the number of nonzero elements in $\mathbf{x}$. There are relatively simple conditions on $A$ that guarantee a feasible solution to (1), however the solution of this problem is combinatorial in nature, and therefore NP-hard.

It has been shown that if $\|\mathbf{x}\|_{0}$ is small [55], the optimal solution to (1) can also be found by solving

$$
\hat{\mathbf{x}}=\min \|\mathbf{x}\|_{1} \text { such that }\|\mathbf{b}-A \mathbf{x}\|_{2}^{2}<\epsilon_{2} .
$$

This is a convex programming problem and can be solved in polynomial time. If $\epsilon_{2}$ is unknown, we can rewrite the above in the following form:

$$
\hat{\mathbf{x}}=\underset{\mathbf{x}}{\arg \min }\left\{\|\mathbf{b}-A \mathbf{x}\|_{2}^{2}+\lambda\|\mathbf{x}\|_{1}\right\}
$$

where $\lambda$ is a weighting factor. The resulting sparsity in $\hat{\mathbf{x}}$ decreases as $\lambda$ increases. The formulation in (2) is now the standard way to frame a "sparse prior" estimation problem and is the core idea behind MIMECS.

\section{METHOD}

The MIMECS method is based on analysis of image patches, which are $p \times q \times r$ 3-D subimages associated with each voxel of the image. Patches are typically small and centered on the voxel of interest-e.g., $p=q=r=3$ or $p=q=r=5$. For convenience we write a patch as the 1 -D vector $\mathbf{b}$ of size $d \times 1$, where $d=p q r$. The voxels within a patch are always ordered in the same way, using a consistent rasterization, in order to create this 1-D representation. Fig. 2 provides an illustrative overview of the MIMECS algorithm.

\section{A. Atlas Description}

We define an atlas as an $(n+1)$-tuple of images, $\left\{a_{1}, a_{2}, \ldots, a_{n}, a_{n+1}\right\}$, where $a_{k}$ has tissue contrast $\mathcal{C}_{k}, k=1, \ldots, n+1$. All atlas images $a_{k}$ are co-registered and sampled on the same voxel locations in space. At each voxel, 3-D patches can be defined on each image and are denoted by the $d \times 1$ vectors $\mathbf{a}_{k}(i)$, where $i=1, \ldots, N$, is an index over the voxels of $a_{k}$. Since $a_{k}$ 's are co-registered, each of them has the exact same number of voxels $N$. The primary aim of MIMECS is to synthesize the $\mathcal{C}_{n+1}$ contrast image using a set of $n$ subject images having contrasts $\mathcal{C}_{1}$ to $\mathcal{C}_{n}$. We define the $\mathcal{C}_{1, n}$ contrast dictionary $A_{1}, A_{1} \in \mathbb{R}^{n d \times N}$, where the $i$ th column of $A_{1}$ is $\mathbf{f}(i)=\left[\mathbf{a}_{1}(i)^{T} \ldots \mathbf{a}_{n}(i)^{T}\right]^{T}$, i.e., an ordered (according to their contrasts $\mathcal{C}_{1}$ through $\mathcal{C}_{n}$ ) column vector composed of patches $\mathbf{a}_{k}(i)$, which is the $i$ th patch from the atlas $a_{k}$. The contrast dictionary $A_{2}, A_{2} \in \mathbb{R}^{d \times N}$, is constructed in a similar manner but only contains the patches found in the atlas $a_{n+1}$, which correspond to the $\mathcal{C}_{n+1}$ tissue contrast. By construction, the $i$ th columns of $A_{1}$ and $A_{2}$ correspond to the same spatial location and represent the $\mathcal{C}_{1}$ through $\mathcal{C}_{n}$ and $\mathcal{C}_{n+1}$ contrasts, respectively.

\section{B. Contrast Synthesis Algorithm}

We assume that there are $n$ subject (input) images $\left\{s_{1}, \ldots, s_{n}\right\}$ available with contrasts $\mathcal{C}_{1}$ to $\mathcal{C}_{n}$. They are co-registered and sampled on the same voxel locations in space. We note that $\left\{s_{1}, \ldots, s_{n}\right\}$ are not registered to $\left\{a_{1}, \ldots, a_{n+1}\right\}$. Also, if there are fewer subject images than the number of images in the atlas, then the atlas contrasts that do not match the subject contrasts are removed, $n$ is reduced (to a minimum of $n=1$ ), and MIMECS is carried out on the available subject images. We first decompose the subject images $s_{k}$ into $d \times 1$ patches $\mathbf{s}_{k}(j)$, where $j$ is an index over the voxels of $s_{k}$. For every $j$, we stack all the patches $\mathbf{s}_{k}(j)$ in a $n d \times 1$ vector $\mathbf{b}_{1}(j)$, in the order of their contrasts $\mathcal{C}_{1}$ through $\mathcal{C}_{n}, \mathbf{b}_{1}(j)=\left[\mathbf{s}_{1}(j)^{T} \ldots \mathbf{s}_{n}(j)^{T}\right]^{T}, \mathbf{b}_{1}(j) \in \mathbb{R}^{n d}$. For simplicity of terminology, we also call any $n d \times 1$ vector a "patch". The aim is to obtain a synthetic $\mathcal{C}_{n+1}$ contrast subject image $\hat{s}_{n+1}$ using $\left\{s_{1}, \ldots, s_{n}\right\}$ and $\left\{a_{1}, \ldots, a_{n+1}\right\}$.

We assume that for a subject image patch $\mathbf{b}_{1}(j)$, a small number of relevant and similar examples can always be found from a rich and overcomplete patch dictionary, $A_{1}$, which has the same contrasts (and assembled into patch vectors in the same order) as $\mathbf{b}_{1}(j)$. It is unlikely that a single patch from $A_{1}$ will perfectly match $\mathbf{b}_{1}(j)$, but it is quite likely that an optimal linear combination of a small number of patches will yield a very close approximation. The problem of finding a few patches to form the linear combination can be solved either by comparing noise characteristics, as derived in nonlocal means [40]-[42], or by assuming the weight vector $\mathbf{x}(j)$ is sparse [44], which is the approach we take here. This leads to the following generic expression for the desired solution:

$$
\mathbf{b}_{1}(j) \approx A_{1} \mathbf{x}(j), \text { for some } \mathbf{x}(j) \in \mathbb{R}^{N},\|\mathbf{x}(j)\|_{0} \ll N, \forall j
$$

where $A_{1}$ is a subset of "relevant" patches taken from the atlas images $\left\{a_{1}, \ldots, a_{n}\right\}$. The relevant patches are chosen according to their $\ell_{2}$ distance from the subject patch, $\mathbf{b}_{1}(j)$, as suggested in [45]. The details are given in Section IV. Once the sparse weight $\mathbf{x}(j)$ is found, the $\mathcal{C}_{n+1}$ contrast subject patch estimate is found by applying the same linear combination to the corresponding $\mathcal{C}_{n+1}$ contrast dictionary, $A_{2}$, i.e.,

$$
\hat{\mathbf{b}}_{2}(j)=A_{2} \mathbf{x}(j) .
$$

The idea of transferring estimated coefficients from one dictionary to another, as we do here, has been explored previously in the context of super-resolution [43] and label fusion [39], [41].

It is important to consider why we seek a sparse representation. The sparsest representation $\mathbf{x}(j)$ is just a single column of $A_{1}$ which is approximately equal to $\mathbf{b}_{1}(j)$. In this case, the corresponding column of $A_{2}$ gives the $\mathcal{C}_{n+1}$ contrast of $\mathbf{b}_{1}(j)$. However, our formulation will not yield unit sparsity in general, but instead a small number of patches from $A_{1}$ whose linear combination gives $\mathbf{b}_{1}(j)$. The motivation behind choosing a 
sparse $\mathbf{x}(j)$ to reconstruct $\mathbf{b}_{1}(j)$ is twofold. First, to reconstruct a subject patch, we want to pick those atlas patches that are close in intensity, i.e., those that are likely to be from the same tissue classes, so it would be undesirable to pick a large number of patches that might tend to mix the tissue classes. Second, if too many similar atlas patches are used to reconstruct $\mathbf{b}_{1}(j)$, then the corresponding $\mathcal{C}_{n+1}$ contrast patch will be overly smooth due to the cumulative effects of small mismatches in each patch. Empirically, we have found that nonsparse $\mathbf{x}(j)$ tends to produce smooth images acting much like a denoising process, while highly sparse $\mathbf{x}(j)$ produces noisy images. In Section IV, an empirical way of choosing the sparsity level is described.

Since the combinatorics of (3) makes it infeasible to solve this problem directly, we use an $\ell_{1}$ minimization strategy and reformulate the problem as

$$
\begin{aligned}
& \hat{\mathbf{x}}(j)=\underset{\mathbf{x} \geq \mathbf{0}}{\arg \min }\left[\left\|\mathbf{b}_{1}(j)-A_{1} \mathbf{x}\right\|_{2}^{2}+\lambda\|\mathbf{x}\|_{1}\right], \forall j, \\
& \text { subject to }\|\mathbf{f}(i)\|_{2}^{2}=1, i=1, \ldots, N
\end{aligned}
$$

where $\mathbf{f}(i)$ denotes the $i$ th column of $A_{1}$, also defined in Section III-A. As in (2), $\lambda$ is a weighting factor that encourages a sparse solution when it is large. Given this optimal combination of the patches in $A_{1}$, which best approximates the subject patch $\mathbf{b}_{1}(j)$, the $\mathcal{C}_{n+1}$ contrast patch is estimated using (4). The full contrast image $\hat{s}_{n+1}$ is reconstructed by taking the union of all central voxels in the reconstructed patches.

There are two key differences in our solution (5) as compared to (2). The first, is the positivity constraint on the coefficients $\mathbf{x}$ in (5). The reason we impose this condition is to encourage the selected patches-i.e., those used in the sparse reconstruction of the subject patch - to have the same "texture" as the subject patch. For example, we do not want a boundary that is GM on the left and WM on the right to be approximated with a negative coefficient multiplying a patch having exactly the opposite orientation. The image being approximated may "look" the same, but the underlying tissues would be wrong and a patch that is synthesized would have incorrect tissues and thus have the wrong appearance. This condition is therefore designed to encourage "gray matter patches" to be used to synthesize "gray matter patches" and so on. We note that computational aspects of this positivity constraint has been previously explored in Lasso [56], and that this constrained $\ell_{1}$ minimization procedure has been shown to be equivalent to an $\ell_{0}$ minimization procedure (like (1) with a similar positivity constraint) if $\mathbf{x}(j)$ is sufficiently sparse [57].

The second difference between (5) and (2) is the requirement that the columns of $A_{1}$ (denoted by $\mathbf{f}(i), i=1, \ldots, N$ ) be normalized to unity. This constraint is necessary in order to guarantee the uniqueness of the solution (by removing the scaling ambiguity), and is a common feature of patch-based techniques in computer vision and image processing [43], [45]. However, unit normalization of patches taken from the atlas MR images, removes the relationship between overall patch intensity and the patch texture, which is essential to distinguish between a "pure" GM patch and a "pure" WM patch, which can only be differentiated by their intensities and not the texture. Therefore, in Section III-C we propose a novel approach that normalizes patches in the $(n d+1)$-dimensional space rather than the $n d$-dimensional space in order to preserve the desired intensity information.

\section{Normalization of $A_{1}$}

To guarantee a unique solution to (5), the columns of $A_{1}$ are normalized such that $\|\mathbf{f}(i)\|_{2}^{2}=1, \forall i=1, \ldots, N$. However, if scale is directly removed from an MR image patch, then a key feature in distinguishing tissue types is lost and patches used in the sparse reconstruction are less likely to be selected from the same tissue type. It is common in the sparse reconstruction literature to use prior training to learn how to select a subset of patches from which to synthesize a given subject patch. In our prior work [47]-[49] we used an atlas selection method based on image classification in order to restrict the dictionary to patches that are likely to come from appropriate tissue classes [47], [48]. We note that the present method supports this strategy since we use a subset $A_{1}$ of patches from which to select a sparse collection (see Section IV). However, an important goal in the present work is to eliminate both the dependency on prior training and the requirement for a classification step prior to synthesis. We have accomplished this using the "trick" of normalizing the patches (e.g., $\mathbf{b}_{1}(j)$ 's or $\mathbf{f}(i)$ 's) in a $(n d+1)$-dimensional space rather than the $n d$-dimensional space, which we now describe.

All the atlas and subject patches are first globally normalized such that their maximum of their norms is unity. Define

$$
m=\max _{i, j}\left\{\|\mathbf{f}(i)\|_{2},\left\|\mathbf{b}_{1}(j)\right\|_{2}\right\} .
$$

Then the patches are scaled as follows:

$$
\begin{aligned}
\tilde{\mathbf{f}}(i) & =\frac{\mathbf{f}(i)}{m} \quad \forall i, \\
\tilde{\mathbf{b}}_{1}(j) & =\frac{\mathbf{b}_{1}(j)}{m}, \quad \forall j .
\end{aligned}
$$

This global scaling guarantees that relative intensities are preserved and that all intensities of both subject and atlas fall in the range $[0,1]$. Now, we project both the subject patch and all atlas patches to the unit sphere in $\mathbb{R}^{n d+1}$ as follows:

$$
\begin{aligned}
\mathbf{f}^{\prime}(i) & =\left[\frac{\tilde{\mathbf{f}}(i)}{\sqrt{1-\|\tilde{\mathbf{f}}(i)\|^{2}}}\right], \quad \forall i \\
\mathbf{b}_{1}^{\prime}(j) & =\left[\frac{\tilde{\mathbf{b}}_{1}(j)}{\sqrt{1-\left\|\tilde{\mathbf{b}}_{1}(j)\right\|^{2}}}\right], \quad \forall j .
\end{aligned}
$$

At this point, both the subject patch and the atlas patches are normalized to unity, satisfying $\left\|\mathbf{f}^{\prime}(i)\right\|_{2}^{2}=1, \forall i$, and $\left\|\mathbf{b}_{1}^{\prime}(j)\right\|_{2}^{2}=$ $1, \forall j$. The normalization criterion in (5) is therefore automatically satisfied if the $\mathcal{C}_{1, n}$ contrast dictionary $A_{1}$ is changed to $A_{1}^{\prime} \in \mathbb{R}^{(n d+1) \times N}$ such that the columns of $A_{1}^{\prime}$ are $\mathbf{f}^{\prime}(i), i=$ $1, \ldots, N$.

Given this new normalization, we rewrite (5) as

$$
\hat{\mathbf{x}}(j)=\underset{\mathbf{x} \geq \mathbf{0}}{\arg \min }\left[\left\|\mathbf{b}_{1}^{\prime}(j)-A_{1}^{\prime} \mathbf{x}\right\|_{2}^{2}+\lambda\|\mathbf{x}\|_{1}\right], \forall j .
$$


The solution of (6) yields a nonnegative combination of the columns of $A_{1}^{\prime}$ that sparsely approximates $\mathbf{b}_{1}^{\prime}(j)$. The resulting reconstructed patch matches both the pattern and intensities within the target patch. We use this solution $\hat{\mathbf{x}}(j) \in \mathbb{R}^{N}$ to synthesize the $\mathcal{C}_{n+1}$ contrast subject patch in $\mathbb{R}^{d}$. This works correctly because we maintain the association of columns in $A_{1}, A_{2}$ and $A_{1}^{\prime}$. Once $\hat{\mathbf{b}}_{2}(j)$ is found from (4), we use only its central value in the reconstructed image $\hat{s}_{n+1}$.

\section{IMPLEMENTATION}

\section{A. Patch Size and $\ell_{1}$ Solvers}

For all the experiments reported in this and subsequent sections, we used patches of dimension $3 \times 3 \times 3$ voxels, i.e., $d=$ 27 . We used two freely available $\ell_{1}$ solver packages, the largescale $\ell_{1}$-regularized least-squares $\left(\ell_{1}\right.$ _ls) [58] and CVX [59], to optimize (6). From our experience, $\ell_{1} \_$ls is the faster of the two, typically having $\sim 1 \mathrm{~ms}$ run-time per patch, while CVX on average takes $\sim 10 \mathrm{~ms}$. However, CVX is more robust, managing to produce reasonable results in cases where $\ell_{1}$ ls does not converge. Thus, we use $\ell_{1} \_$ls as our $\ell_{1}$ solver except in the following three cases.

1) The algorithm does not converge and gives null output.

2) The output $\hat{\mathbf{x}}(j)$ contains a complex number.

3) Experimentally we have found that for typical SPGR images, $\|\hat{\mathbf{x}}(j)\|_{1}$ has a Laplacian distribution [60], [61] with mean 1 and small variance $(\sim 0.005)$. Thus, if $\|\hat{\mathbf{x}}(j)\|_{1} \gg$ 1 for some $j$, the algorithm is assumed to converge to an undesirable solution.

For these cases, $\ell_{1}$ ls having failed, we use CVX.

\section{B. Dictionary Selection}

A typical $256 \times 256 \times 199$ isotropic MR image, with voxels of size $1 \mathrm{~mm}^{3}$, contains about one million nonbackground patches. It is computationally intractable to solve (6) with all such patches included in the dictionary $A_{1}^{\prime}$. Recall that for $3 \times 3 \times 3$ patches, our dictionary $A_{1}^{\prime} \in \mathbb{R}^{28 \times N}$, could potentially be a $28 \times 1000000$ matrix, if $N$ is assumed to be indexing over all patches in the atlas image $a_{1}$. To avoid the computational bottleneck of large $N$ we construct a dictionary for each patch $\mathbf{b}_{1}^{\prime}(j)$, that is of size $N=100$. This is done by selecting 100 patches from the global collection of one million patches, thus drastically reducing run time. In keeping with the literature [45], we construct our patch dictionary, $A_{1}^{\prime}$, to consist of only those atlas patches that are close to $\mathbf{b}_{1}^{\prime}(j)$ in an $\ell_{2}$ sense. It is also in accordance with the assumption that the atlas patches should be close to the subject patch. To achieve this in our setting, we sort the atlas patches, $\mathbf{f}_{1}^{\prime}(i), i=1,2, \ldots, N$, by their $\ell_{2}$ distance to the current patch, $\mathbf{b}_{1}^{\prime}(j)$. The nearest 100 patches, $\mathbf{f}_{1}^{\prime}(i), i=1,2, \ldots, 100$, are then used to generate the dictionary, $A_{1}^{\prime}$, for the current patch, then $\hat{\mathbf{x}}(j)$ is found using (6). To accelerate the search for the 100 nearest patches, we use a k-d tree [62]. We construct $A_{2}^{\prime}$ as the corresponding $\mathcal{C}_{2}$ contrast patches for the collection $\mathbf{a}_{2}(i), i=1,2, \ldots, 100$. Our selection of $N=100$ is based on our experience, having observed that smaller $N$ does not always yield the best candidate patches and larger $N$ generally shows little improvement in the reconstruction result.
TABLE I

Fraction of Atlas Patches Used, Averaged Over All SubJeCt PATChes From a $256 \times 256 \times 199$ IMAGE, From EACH OF CEREBrospinal Fluid (CSF), Gray MATTER (GM), White MATTER (WM), AND THE VENTRICLES (VEN)

\begin{tabular}{llllll}
\hline & & \multicolumn{4}{c}{ ATLAS } \\
& & CSF & VEN & GM & WM \\
\hline \multirow{4}{*}{ SUBJECT } & CSF & 0.71 & 0.15 & 0.14 & 0.00 \\
& VEN & 0.16 & 0.78 & 0.06 & 0.00 \\
& GM & 0.10 & 0.03 & 0.77 & 0.10 \\
& WM & 0.00 & 0.00 & 0.23 & 0.77 \\
\hline
\end{tabular}

To demonstrate the validity of the choice of atlas patches using such an $\ell_{2}$ proximity criteria between them, Table I shows the fractions of major atlas tissues used to reconstruct a subject patch, averaged over all subject patches from a $256 \times 256 \times 199$ real SPGR image, shown in Fig. 3. The classification of the center voxel of a patch is used to classify the patch. Ideally, the table should contain 1 at the diagonal entries with 0 otherwise. From the table, $78 \%$ of the atlas patches, that are used to reconstruct the subject ventricle patches are from atlas ventricle patches, while the remainder are from atlas CSF $(16 \%)$ and GM $(6 \%)$. However, we have observed that the nonzero fraction of atlas GM patches used to synthesize subject ventricles can be attributed to the partial volume effect at the boundary of WM and ventricles, which is reconstructed by GM patches. Similarly, the nonzero contributions of atlas CSF and WM patches $(10 \%)$ to reconstruct subject GM patches can be attributed to the partial volume near CSF-GM and GM-WM boundary. On the other hand, the zero contribution of atlas WM patches to reconstruct subject CSF patches or atlas CSF patches for subject WM patches indicate that the tissues are not "interchanged" while reconstructing, which provides evidence that the $(d+1)$-dimensional normalization and the coefficient positivity aspects of the algorithm, as described in Sections III-B and III-C are working as desired.

\section{Sparsity Regularization Coefficient}

The sparsity regularization term, $\lambda$, is an important parameter in our algorithm as it is used for tuning the sparsity of the reconstruction. Through empirical experiments involving hundreds of thousands of patch reconstructions, we have found the algorithm itself to be very stable for $\lambda \in[0,0.85]$; but the quality of reconstruction varies within this range. To get a quantitative sense of the synthesis, we experimentally assessed how the error in synthesis is affected by the choice of $\lambda$. Accordingly, we created an atlas pair $\left\{a_{1}, a_{2}\right\}$ using patches from one part (several contiguous slices) of a subject's SPGR $\left(\mathcal{C}_{1}\right)$ and MPRAGE $\left(\mathcal{C}_{2}\right)$ and then synthesized other slices of the MPRAGE image from the SPGR. Shown in Fig. 3 are three examples of reconstructed MPRAGEs using different values of $\lambda$ and a graph of the root mean square error between the synthesized MIMECS MPRAGEs and the true MPRAGE. We conclude from this experiment that values of $\lambda$ in the range $[0.05,0.85]$ are visually acceptable and produce similar error levels. However, since our original goal was to recover a solution that satisfies an $\ell_{0}$ criterion, we choose to use a larger $\lambda$ from this range because 

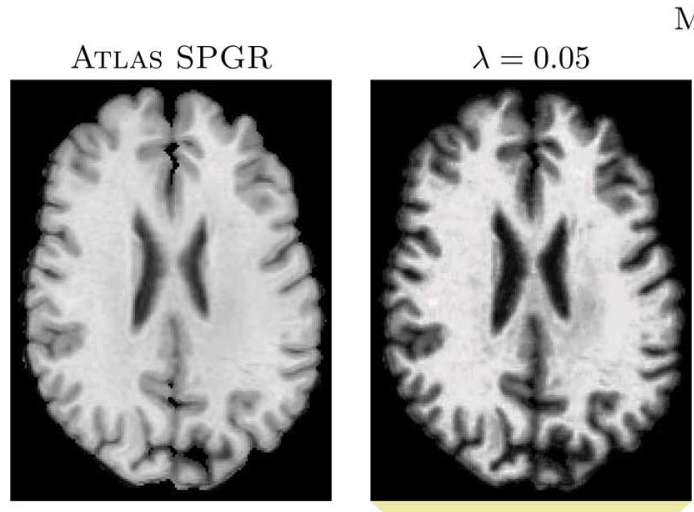

MIMECS RECONSTRUCTIONS
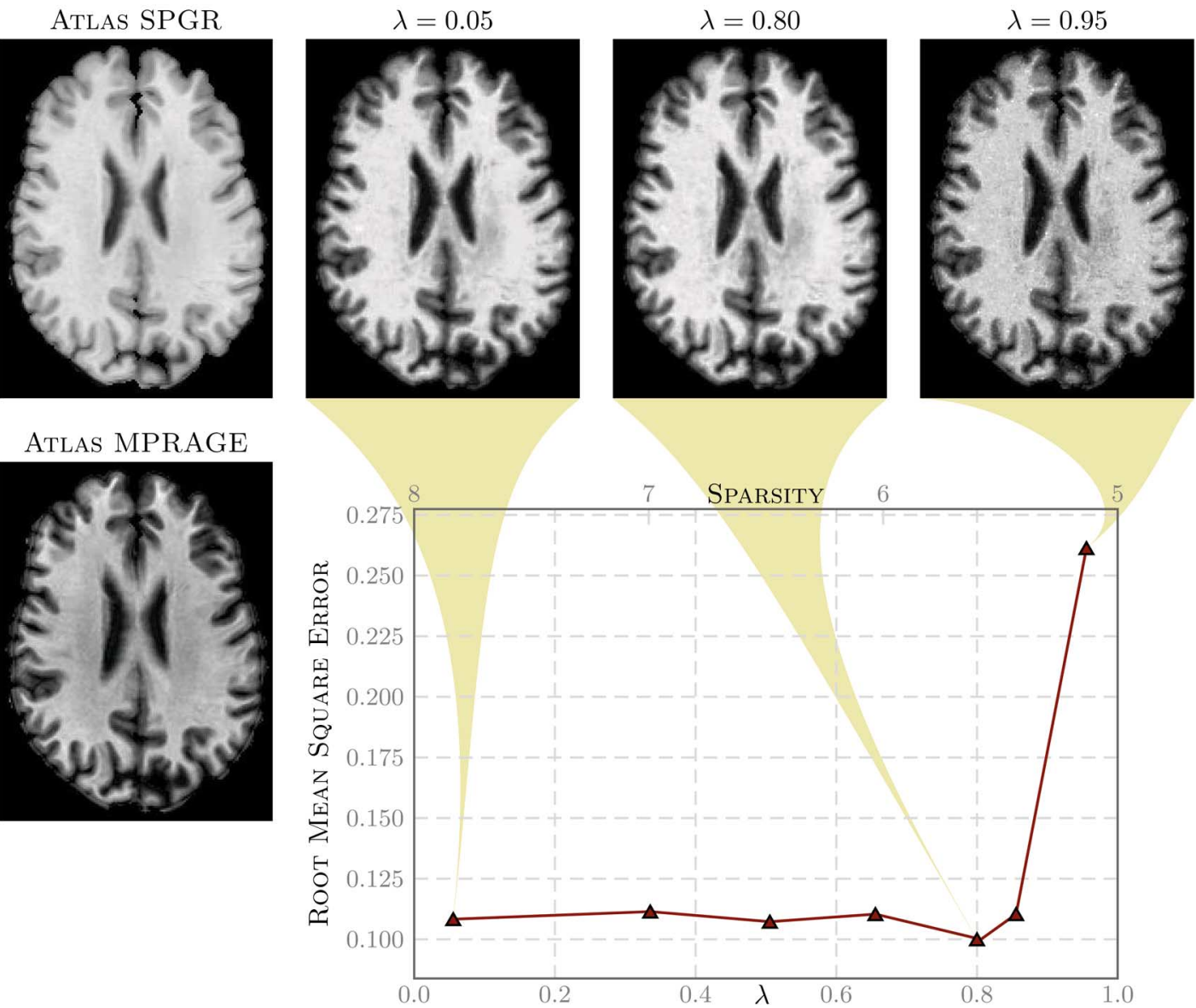

Fig. 3. Effect of sparsity of $\mathbf{x}(j)$ on the $\mathcal{C}_{2}$ contrast: Left most column shows a subject's SPGR (top) and MPRAGE (bottom) acquisitions. Second, third, and fourth columns of the top row show synthetic MPRAGEs generated using another portion of the subject's MPRAGE as the atlas. Synthetic MPRAGEs were generated using $\lambda$ values of $0.05,0.80$, and 0.95 , respectively. Plot shows the MPRAGE synthesis error versus the average sparsity of all $\mathbf{x}(j)$ 's, averaged over all nonzero voxels. Average sparsity scale is on the top of the plot while the sparsity regularization parameter, $\lambda$, is plotted on the bottom axis.

sparser solutions produced by $\ell_{1}$ minimization are more likely to agree with the desired $\ell_{0}$ solution [55]. We have therefore used $\lambda=0.80$ in all of our experimental results reported below.

\section{REsults}

In order to verify the correctness of our MIMECS software implementation, to demonstrate its most basic behavior, and to compare it to histogram matching methods, we first made extensive use of the Brainweb phantom data [63], the Kirby-21 reproducibility data [64], and the BIRN traveling subject data [18]. These results are omitted here for paper length considerations, but they can be found in [65]. The most important results from this set of experiments can be summarized as follows. First, we found that features and noise that are present in the subject $s_{1}$ image but not in the atlas $a_{1}$ image are suppressed to some degree in the reconstruction. This was true both in recovering the same tissue contrast (a kind of "sanity check") and in recovering different tissue contrasts. This observation suggests that MIMECS can be used for noise suppression (though other methods such as nonlocal means might be better) and also serves as a caution that the MIMECS atlas should contain a patch dictionary that is rich enough to encompass the patches that are expected to be found in the source image. Second, the experiments confirmed that sparsity is important when reconstructing patches with significant detail because otherwise the detail will be smoothed out. Third, MIMECS is fairly robust to the specifics of the subject pulse sequence. In particular, we found that an atlas comprising both an SPGR and MPRAGE can be used to synthesize either an MPRAGE or SPGR image even if the subject image did not precisely match the SPGR or MPRAGE pulse sequence parameters of the atlas. Fourth, we found that MIMECS normalization is comparable or better than histogram matching when normalizing the same tissue contrast (e.g., T1-w from T1-w) and, of course, is far more general in that it can recover missing contrasts. Finally, we found that errors in MIMECS synthesis do not take on specific anatomical features, but instead resemble a typical noise pattern of MR images. This was true in both normalization of the same contrasts and synthesis of alternate contrasts. We note that most of the above observations will be evident in the results below, which is another reason why we did not include these results here.

In this section, we focus on potential uses of MIMECS in clinical and scientific studies rather than focusing solely on performance-oriented criteria (since we have previously reported such results in [47]-[49], [65]). Accordingly, each result should be thought of as a "case study" designed to demonstrate a potential 
use. Some studies provide numerical results that demonstrate improvement over the state of the art, while others only show compelling visual results. One case study is cautionary, demonstrating a limitation of MIMECS in a potential application.

The results presented here all involve real, not simulated, MR images. Using a standard preprocessing pipeline for neuroimages, the images were skull-stripped [66], [67], corrected for intensity inhomogeneities using N3 [68], and then normalized using a global linear scaling such that the mode of WM intensities were at unity [28]. The MIMECS algorithm, applied to a $256 \times 256 \times 199$ image, takes $1-2 \mathrm{~h}$ on a 12 -core $2.7 \mathrm{GHz}$ Linux machine using MATLAB (2012a, The MathWorks, Natick, MA, USA). The bulk of the computational time is spent in the $\ell_{1}$ solver.

\section{A. Longitudinal Analysis of Multi-Contrast Data}

In large longitudinal studies (such as the BLSA [19] or ADNI [20]), the scanner and/or acquisition protocol can change over time. An example of such phenomenon is observed in the BLSA data set where 14 scans of a normal subject were acquired in consecutive years. The first 11 scans were acquired using an SPGR protocol on a GE 1.5T scanner, while the last three scans were acquired using an MPRAGE protocol on a Philips 3T scanner. It is noted in [69] that it is difficult to analyze these data consistently. There has been several recent publications trying to address this problem [70]-[72].

To account for the change in acquisition protocol, we used MIMECS to synthesize images having SPGR contrasts from the three MPRAGE scans of one BLSA subject. A subject from the BIRN traveling subject database [18] having both SPGR and MPRAGE scans was used as the atlas. Fig. 4 shows real and synthetic images from this subject. The MIMECS images clearly display the SPGR tissue contrast, which is observed to be very similar to the earlier time frames. The synthetic images are visibly less noisy and slightly blurrier than the original SPGR and MPRAGE images.

Since analysis of longitudinal changes in brain volume is the primary objective in the BLSA study (cf. [12]), we used a state-of-the-art longitudinal segmentation algorithm called longitudinal FreeSurfer [71] (longFS) to segment and label the resulting data. LongFS can only be applied to data having the same pulse sequence (either SPGR or MPRAGE), so we were able to apply it to 14 volumes comprising the original 11 SPGR volumes and the three MIMECS SPGR volumes. Plots showing the computed GM and ventricle volumes as a function of subject age are shown in Fig. 5 (red with asterisks). For comparison, we applied longFS to each of the longitudinal segments acquired using the same pulse sequence. Accordingly, Fig. 5 also shows the GM and ventricle volumes for the 11 time frames having SPGR (blue with triangles) data and the three time frames from both the original MPRAGE (using the -mprage flag in longFS) and the MIMECS SPGRs (pink with crosses and green with squares, respectively).

The first key observation to make from Fig. 5 is that longFS yields very different segmentation results and corresponding volume measurements given source data with different pulse sequences. Every neuroimage segmentation algorithm that we have ever tested shares this discrepancy, so this observation

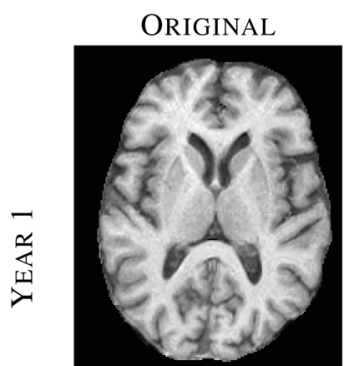

SYNTHETIC
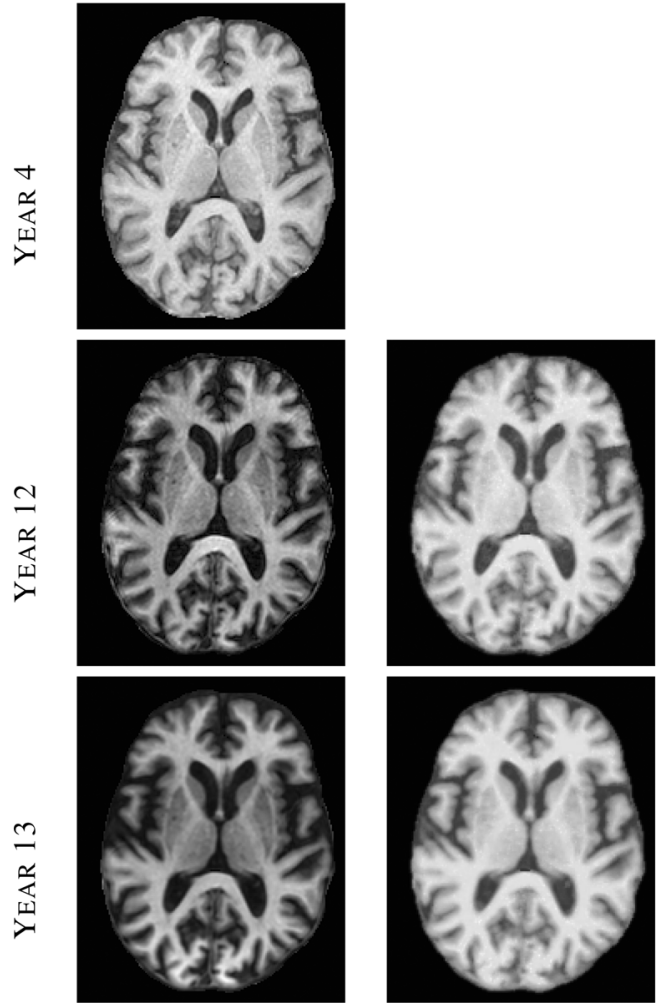

Fig. 4. Left column shows images from four (out of 14) time-points of a normal BLSA subject, where each image was acquired approximately one year apart. Right column shows synthetic SPGR images, synthesized using MIMECS applied to the corresponding MPRAGE images in the left column.

should not be interpreted as an indictment of longFS. The second key observation is that MIMECS synthesis permits longFS to make very consistent longitudinal segmentations (at least as far as the volume measured from these segmentations) across the entire collection. Even the segmentation results from the short sequence of synthetic SPGRs are more consistent with the older data. Therefore, we conclude that MIMECS may be a useful preprocessing step prior to analysis of volume changes in longitudinal neuroimaging studies.

\section{B. Average Atlas Construction}

While developing voxel-based measures from a population, it is common practice to register all the images to an average atlas space. However, pooling data from two populations can be difficult when individual images from two populations are acquired with different pulse sequences or on heterogeneous scanners. In this case study, we show that by synthesizing contrasts using MIMECS, a better average atlas can be created while pooling subjects from two different data sets. This MIMECS case study 

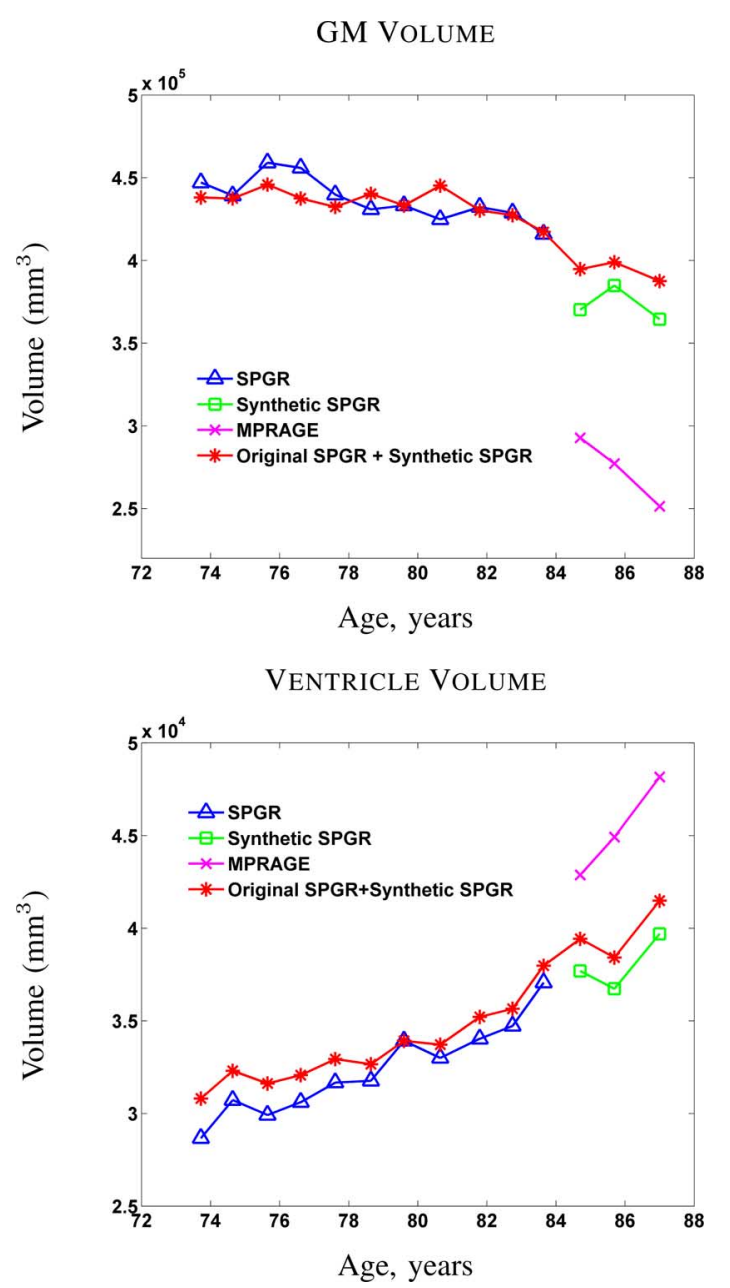

Fig. 5. GM and ventricle volumes from longitudinal FreeSurfer [71] of a normal subject with 14 scans (first 11 are SPGR, last three are MPRAGE).

represents a new capability; the use of multiple data sources in atlas construction enabling richer and more complete analyses.

As a straight forward demonstration of this, we selected five normal subjects from the BLSA database (1.5T GE, SPGR) and five normal subjects from the OASIS [73] databases (1.5T Siemens, MPRAGE), all male and right-handed with age range 60-70 years. A state-of-the-art registration tool SyN [7] was used to create average atlases from these 10 subjects. A crosssection and zoomed region of this result are shown in Fig. 6(a) and (b). We then synthesized volumes with MPRAGE contrast from the five BLSA subjects and created an average atlas from the 10 MPRAGE contrast images. This result and a zoomed region are shown in Fig. 6(c) and (d). Visually, the atlas created from all MPRAGE contrasts has sharper features than the one with the mixture of SPGR and MPRAGE contrasts [compare Fig. 6(b) and (d)].

To show a quantitative improvement in atlas quality, Fig. 6(e) and (f) shows the voxel-wise standard deviations of intensities from the 10 subjects used to create each of the atlases (after deforming them into the atlas space). It can be seen that the standard deviations are much smaller when synthetic MPRAGEs are combined with OASIS MPRAGEs, especially around the ventricles and in the GM, indicating better feature matching in

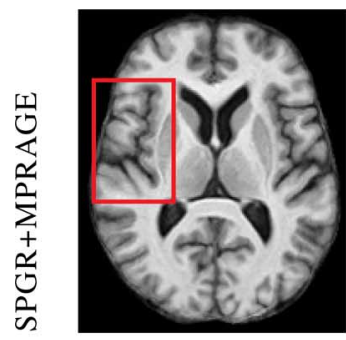

(a)

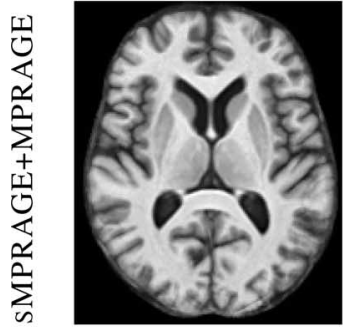

(c)

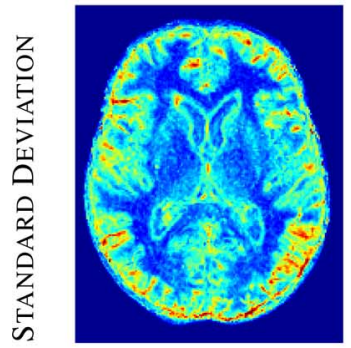

(e)

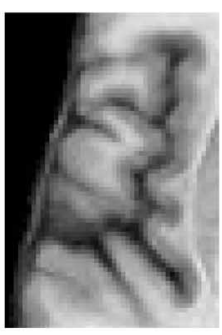

(b)

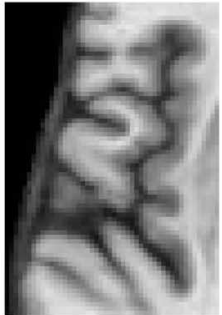

(d)

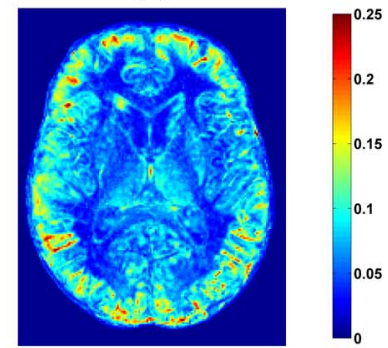

(f)
Fig. 6. (a) Average atlas created using SyN from five BLSA (SPGR) and five OASIS (MPRAGE) images and (b) a zoomed region. (c) Average atlas from five synthetic MPRAGEs from BLSA and five OASIS images (MPRAGE) and (d) a zoomed region. (e) Intensity standard deviation image from SPGR+MPRAGE atlas and (f) intensity standard deviation image from sMPRAGE+MPRAGE atlas.

registration. We then segmented the two atlases using TOADS [2]. A t-test shows that the average standard deviations of CSF, ventricles and GM are significantly lower $(p$-value $<0.01)$ for the atlas obtained with all MPRAGE contrasts compared to the one obtained with the mixture of SPGR and MPRAGE contrasts. This average atlas construction case study represents an enhancement to existing technologies, which would provide for richer atlas construction and perhaps more accurate deformation fields carrying each subject into the average atlas space (and vice versa).

\section{Segmentation Bias in Cross-Sectional Analysis}

When pooling data from different studies, the choice of scanning protocols introduces algorithm bias in the quantification even the simplest biomarkers, such as GM and WM volume. To study this bias we used 42 age-matched normal subjects (male, right-handed, and 60-70 years of age), 21 from the BLSA database (all SPGR scans) and 21 from the OASIS [73] database (all MPRAGE scans). The images were then skull-stripped [67] and segmented with FreeSurfer [74]. Plots of average ventricle and brain $(\mathrm{GM}+\mathrm{WM})$ volume are shown in Fig. 7. It is known that FreeSurfer generally overestimates brain volume when using SPGR data [75], and we observe this same tendency here [Fig. 7(a)]. To test the null hypothesis that volumes 


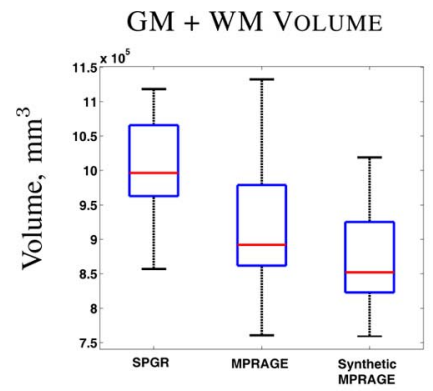

(a)

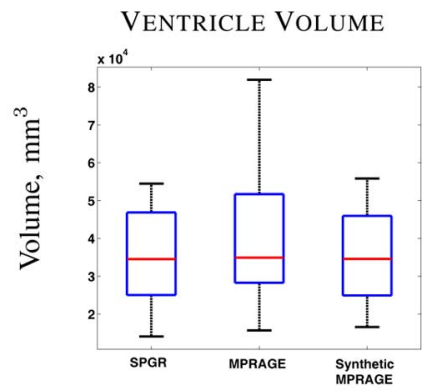

(b)

Fig. 7. (a) Brain volume $(\mathrm{GM}+\mathrm{WM})$ and (b) ventricle volume computed using FreeSurfer [74] on 21 normal subjects from the BLSA (SPGR) database and 21 normal subjects from the OASIS (MPRAGE) database, as well as on the 21 synthetic MPRAGEs generated from the BLSA SPGR images.

for each structure are the same, we use a Wilcoxon rank-sum test with a significance level of 0.01 . The average brain volumes $(\mathrm{GM}+\mathrm{WM})$ computed from the SPGR scans are significantly higher $(p$-value $<0.01)$ than the MPRAGE brain volumes. There was no statistically significant difference for the ventricle volumes for the two populations.

We then used MIMECS (with a BIRN SPGR/MPRAGE atlas, as above) to synthesize MPRAGE contrasts from the SPGR scans and then used FreeSurfer to segment the synthetic scans. As shown in Fig. 7(a), the brain volumes computed this way are comparable to those computed using OASIS/MPRAGE data; the same Wilcoxon rank-sum test shows that the MPRAGE and synthetic MPRAGE come from the same distribution. We also observe that the ventricle volumes do not change in a statistically significant way after synthesis, again, with respect to a Wilcoxon rank-sum test. This case study shows that MIMECS can remove the systemic bias in volumetric analysis arising from scanner and pulse sequence differences in studies that pool multi-site and multi-center data.

\section{Distortion Correction in $b_{0}$ Scans}

Diffusion MR images are acquired using echo-planar techniques and are geometrically distorted by susceptibility and eddy currents unless corrected [76]. Although eddy current distortion correction can be carried out by consideration of the MR physics and pulse sequence alone, susceptibility causes subject-dependent distortion and is generally carried out retrospectively by deformably registering the $b_{0}$ image to a structural image such as a T1-w or T2-w image [77]. The $b_{0}$ image - a minimally-weighted diffusion image - has a tissue contrast similar to that of a conventional T2-w spin-echo image, thus making T2-w images the preferred target for distortion correction. But what happens if no T2-w image was acquired as part of the protocol? In this case, the correction might be attempted by registering the $b_{0}$ image to the T1-w image, leading generally to an inferior result. Here, we consider an alternative approach using MIMECS.

Fig. 8(c) and (e) shows an MPRAGE image and a $b_{0}$ image, respectively, acquired in the same imaging session of a subject from the Kirby-21 database [64]. The $b_{0}$ image shows significant distortion in the anterior portion of the brain. Fig. $8(\mathrm{~g})$ shows the $b_{0}$ image after it has been registered to the MPRAGE

image using SyN [7] (with mutual information as the similarity metric). By comparing the GM/WM boundary generated by FreeSurfer segmentation of the MPRAGE [see Fig. 8(f)] to the underlying intensities of the registered $b_{0}$ image [see Fig. 8(h)], it is clear that the correction is inadequate.

Given the atlas shown in Fig. 8(a) and (b), we used MIMECS to synthesize T2-w image from the MPRAGE image; the result is shown in Fig. 8(d). We then registered the $b_{0}$ image to the synthetic T2-w using SyN (with the cross-correlation similarity metric), yielding the distortion-corrected $b_{0}$ image shown in Fig. 8(i). The red arrow in Fig. 8(i) indicates an area of improvement, which can be visually confirmed on the zoomed region containing the GM/WM contour shown in Fig. 8(j).

This case study demonstrates a potential use of MIMECS in cases where data is missing. It also reveals a new potential approach to registration of multimodal data, wherein synthesis of the alternate tissue contrast is used with a cross-correlation metric rather than the conventional mutual information metric applied to different tissue contrasts. This concept could have far more general applicability than the simple case example demonstrated here.

\section{E. High Resolution T2-W Synthesis}

Because of time constraints, T2-w scans are often acquired at lower resolution than T1-w scans. If high-resolution (hi-res) T2-w scans were available, however, they could be used for many purposes including lesion segmentation [78], [79] and $b_{0}$ dewarping (as described in the previous section). Super-resolution techniques using nonlocal means have previously been proposed to synthesize hi-res T2-w images [38], [80]. These methods use patches from both the hi-res T1-w image and a lo-res T2-w image and index into an atlas comprising these two images and a hi-res T2-w image.

MIMECS provides an alternate way to carry out super-resolution, where the hi-res T2-w image can be synthesized directly from a subject's high-res T1-w image. This means that acquisition of the lo-res T2-w image is unnecessary (for image processing purposes). As an example, consider the SPGR and T2-w images shown in Fig. 9(a) and (b), respectively. Both data sets were acquired axially with native resolutions of $0.94 \times 0.94 \times 1.5 \mathrm{~mm}$ and $0.94 \times 0.94 \times 5 \mathrm{~mm}$, so the low through-plane resolutions are readily apparent as vertical blurring in both of these coronal images - and, clearly, it is much worse in the T2-w image. We use Brainweb [63] T1-W and T2-W images as the atlas images, $a_{1}$ and $a_{2}$, as shown in Fig. 9(c) and (d), because both are available at high resolution. MIMECS is then used to synthesize a hi-res T2-w image from the SPGR image alone, and the result is shown in Fig. 9(f). For comparison, an upsampled (super-res) result using the nonlocal means method of [80] is shown in Fig. 9(e). It is readily apparent by visual inspection that MIMECS produces a superior hi-res T2-w image over both the original T2-w acquisition and the nonlocal means result.

\section{F. MIMECS Image Segmentation}

In this section, we show that MIMECS can also be used as a tissue classification and image segmentation method. In this experiment, we use five atlases, each containing a T1-W 

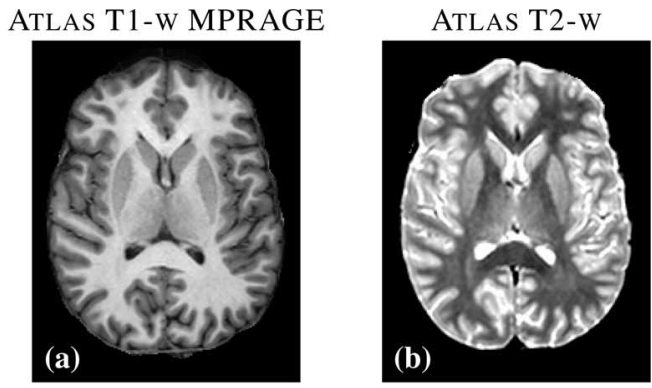

SUBJECT T1-W MPRAGE
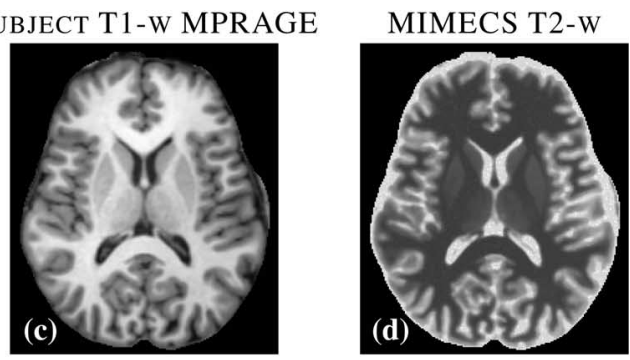

T1-W CONTOURS

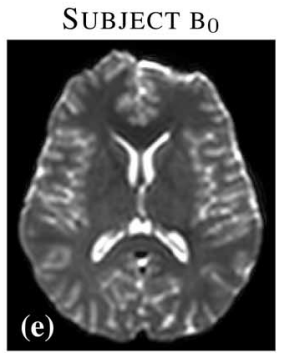

T1-W CORRECTED

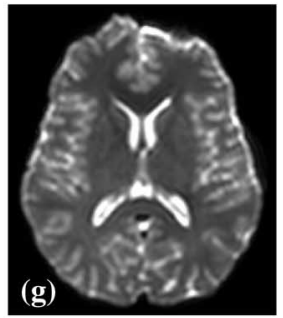

MIMECS CORRECTED

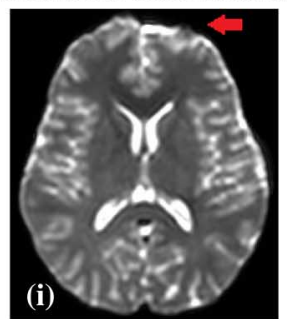

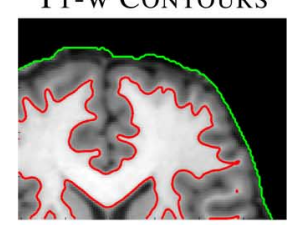

(f)

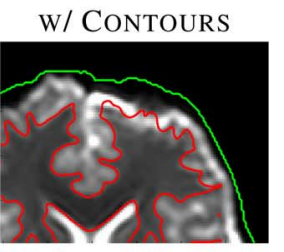

(h)

W/ CONTOURS

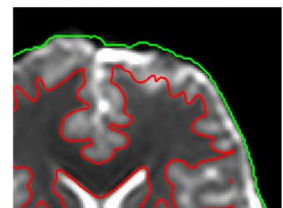

(j)

Fig. 8. Images (a) and (b) show an atlas pair consisting of MPRAGE and T2-w scans of a normal subject from the Kirby-21 data set. A subject MPRAGE scan (c) is used with MIMECS to synthesize a T2-w image of the subject (d). The acquired subject $b_{0}$ image (e) is deformably registered to the subject T1-w image using SyN (with the MI criterion) yielding the corrected image (g). Contours generated from FreeSurfer on the T1-w image (f) are shown on the geometry corrected $b_{0}$ image (h). The acquired $b_{0}$ image is deformably registered to the synthetic MIMECS image using SyN (CC criterion) to create a MIMECS corrected image (i). Overlaid contours on this image (j) reveal much better geometry correction.

SPGR images and its segmentation into CSF, GM, and WM tissue classes. The segmentations, illustrated in the top center

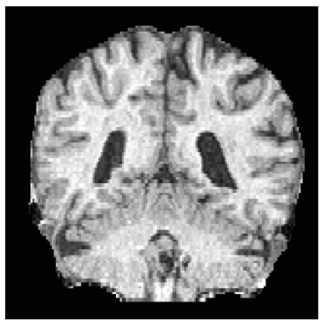

(a)

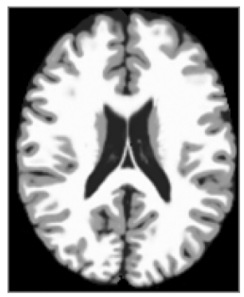

(c)

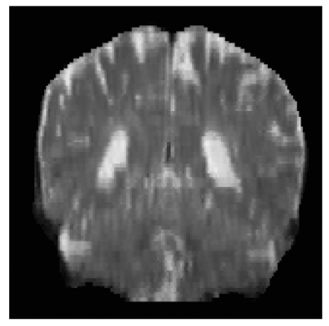

(e)

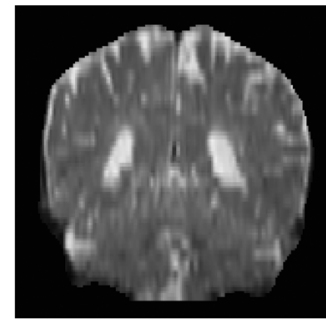

(b)

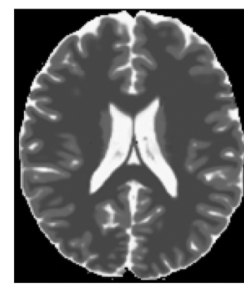

(d)

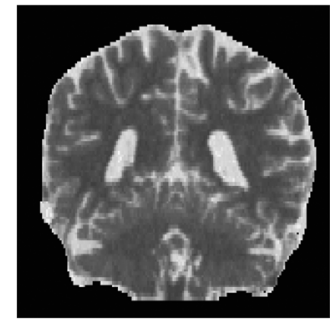

(f)
Fig. 9. Coronal views of (a) SPGR (native resolution $0.94 \times 0.94 \times 1.5 \mathrm{~mm}$ ) and (b) T2-w (native resolution $0.94 \times 0.94 \times 5 \mathrm{~mm}$ ) scans of a subject. (The T2-w image has been upsampled to the SPGR resolution using trilinear interpolation.) Brainweb (c) T1-w and (d) T2-w phantoms used as an atlas in MIMECS. (e) A hi-res T2-w image upsampled using a nonlocal super-resolution method. (f) MIMECS synthesized hi-res T2-w image.

of Fig. 10, were carried out by FreeSurfer [74] followed by expert manual corrections.

In order to make use of MIMECS when label images rather than image intensities are involved, we must rethink its steps. Consider a single atlas (an image and its segmentation) and a single voxel (with its corresponding subject patch) in the MIMECS procedure (as described in Section III-B). Determination of the sparse coefficient vector $\hat{\mathbf{x}}(j)$, which defines an optimal combination of atlas patches that best match the subject patch [see (5)], can be carried out as usual. But to use this vector to form a linear combination of label patches as in (4) does not make sense since the labels are discrete quantities. Two simple modifications are needed to solve this problem.

First, we form the contrast dictionary $A_{2}$ as usual; it comprises a single patch in each column and each patch is a collection of labels from $a_{2}$. Let us represent the labels as follows: CSF $(k=1), \operatorname{GM}(k=2)$, and WM $(k=3)$. We now form a vector contrast dictionary $\mathbf{A}_{2}=\left(A_{2}^{1}, A_{2}^{2}, A_{2}^{3}\right)$ with its elements defined by

$$
A_{2}^{k}=\left\{\begin{array}{ll}
1, & \text { if } A_{2}=k \\
0, & \text { otherwise }
\end{array} \quad k=1,2,3 .\right.
$$

This process is equivalent to viewing the $a_{2}$ dictionary image as a vector membership function rather than a label image. The 


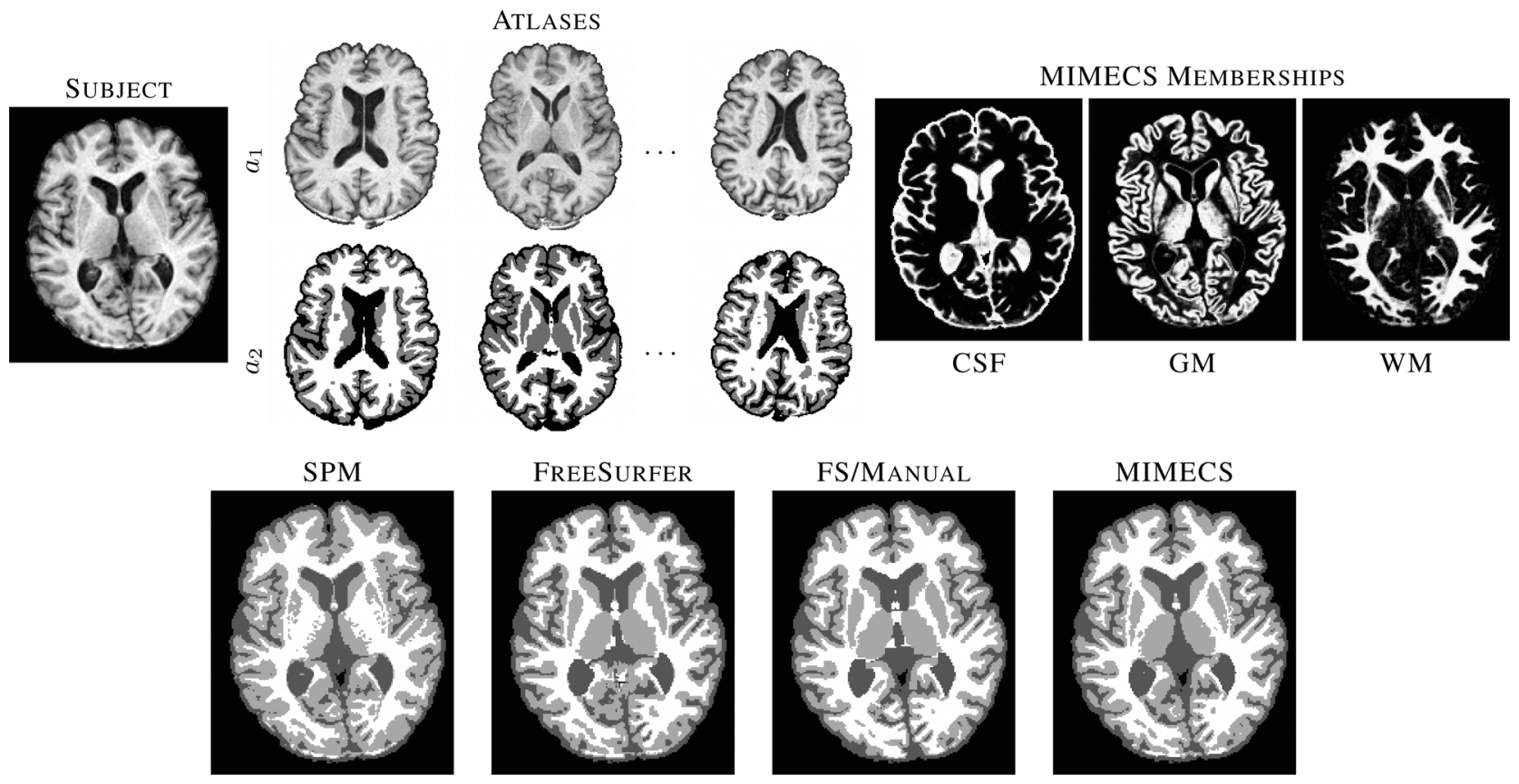

Fig. 10. Top row shows a subject T1-w SPGR image, three of the five classification atlases, and fuzzy memberships produced by MIMECS-based tissue classification. Hard segmentations from two leading automatic methods and a manually-corrected method are compared to the hard segmentation of MIMECS.

elements of this dictionary are now interpreted as vector membership functions and we are free to form convex combinations of these vectors in order to produce new (fuzzy) membership functions.

This leads us to the second modification of the usual MIMECS process. It is desirable for the linear combination of vector membership functions to be convex combinations; in this way the elements of the resultant membership function will add up to unity and can also be viewed as either probabilities or partial fractions of the tissue classes. Since the elements of $\hat{\mathbf{x}}(j)$ are nonnegative, we can guarantee its use in making a convex combination by simply dividing the inner products by the sum of the elements of $\hat{\mathbf{x}}(j)$. Accordingly, we form a MIMECS-based membership function as follows:

$$
\mu_{k}(j)=\frac{A_{2}^{k} \hat{\mathbf{x}}(j)}{\|\hat{\mathbf{x}}(j)\|_{1}}, \quad k=1,2,3 .
$$

The result of this whole process is a fuzzy tissue classification at each voxel and therefore a fuzzy (or soft) segmentation of the subject image. We have found that the result produced with just one atlas is not highly accurate. To produce a more robust result, we apply this exact approach to five atlases and average the resulting membership functions at each voxel. To produce a hard classification - as in the segmentations that are used in the atlas-we use the conventional maximum membership criterion.

An example of the MIMECS tissue segmentation process is shown in Fig. 10. Note that the membership functions (upper right) look very much like the soft segmentations produced by fuzzy C-means or mixture model methods; they can be used exactly as one might use such functions in subsequent segmentation or analysis stages. A hard segmentation is shown on the bottom right. Hard segmentations from SPM, fully-automatic
FreeSurfer (with default parameters), and manually-corrected FreeSurfer are shown for comparison (see bottom row). The MIMECS segmentation has many desirable visual features and compares quite favorably to the manually-assisted FreeSurfer result.

For quantitative comparison, Dice coefficients for the SPM, fully-automatic FreeSurfer, and MIMECS results against the manually-corrected FreeSurfer result were computed, yielding $0.8630,0.8811,0.8837$, respectively. MIMECS shows a small improvement in this example. The most important point to be made is that this MIMECS segmentation approach does not rely on any statistical (spatial or intensity) prior. Instead it obtains its result through the use of dictionary examples alone. Similar methods in the literature (e.g., [39], [81]-[83]) are gaining wide support because of their superior performance and generalizability in comparison to model-based methods.

\section{G. FLAIR Synthesis}

FLAIR (fluid attenuated inversion recovery) images have become important in detecting lesions in the brain, especially in WM. Many older studies did not use the FLAIR pulse sequence, so image processing algorithms designed to exploit the FLAIR tissue contrast cannot be directly applied. Also, since the FLAIR pulse sequence is very sensitive to patient motion some FLAIR images are "spoiled" by motion artifacts and are also not amenable to image processing algorithms. We therefore carried out a study on the use MIMECS to synthesize FLAIR images.

The atlas we used for this experiment has three images: $a_{1}$ has an MPRAGE contrast, $a_{2}$ has a T2-w contrast, and $a_{3}$ has a FLAIR contrast. Cross-section of the atlas images are shown in the left-hand column of Fig. 11. The goal is to apply MIMECS using two subject images - an MPRAGE image and a T2-w 

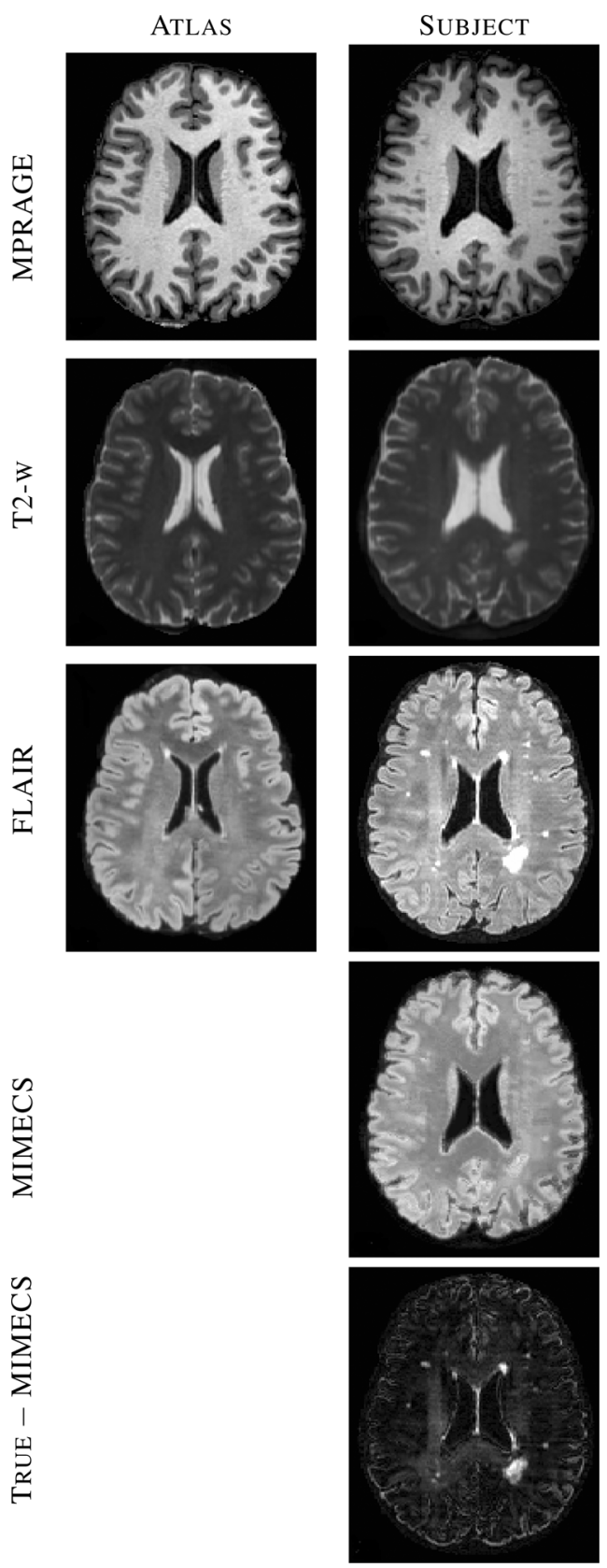

Fig. 11. Left-hand column contains an atlas for FLAIR synthesis. Right-hand column shows the subject's true images, a synthetic MIMECS FLAIR image, and a subtraction image (true FLAIR minus synthetic MIMECS FLAIR).

image - to synthesize a FLAIR image. Both the atlas and the subject has WM lesions, so the presence of a unique lesion patch signature in the subject should also be present in the atlas.

Cross sections of subject images and the synthetic MIMECS FLAIR image are shown in the right-hand column of Fig. 11. The true FLAIR image for this subject, which is not used in the synthesis algorithm, is shown for comparison. It is immediately evident that the lesions in the synthetic FLAIR image have been rendered as if they are GM-i.e., they are not bright like lesions in true FLAIR images. It is relatively easy to understand why this happens. If one visually identifies a lesion in the (atlas or subject) FLAIR image, it is readily apparent that these same regions on both the MPRAGE and T2-w have intensities that are similar to GM within their respective tissue contrasts. Thus, patches involving lesions in both MPRAGE and T2-w are indistinguishable from patches involving GM.

This experiment reveals a limitation of MIMECS synthesis. Stated simply, the set of pulse sequences used to synthesize a new image must contain intensity signatures that can uniquely identify the tissue classes that are present in the tissue contrast to be synthesized. This is certainly a cautionary note about MIMECS synthesis and it deserves further study to determine its specific limitations. On the other hand, the ability to synthesize images devoid of lesions presents an opportunity to enhance lesions by subtraction. The bottom right image in Fig. 11 is a subtraction image (true subject FLAIR minus synthetic FLAIR) that shows potential for visual enhancement of lesions or for computational assistance in finding lesions.

\section{SUMMARY AND DisCUSSION}

MIMECS is a new approach to image intensity normalization and tissue contrast synthesis. It is based on sparse dictionary reconstruction methods with a novel patch normalization approach that makes dictionary selection straightforward. For each voxel, a dictionary is found by casting all patches into one higher dimension and searching for "like" patches using a rapid kd-tree search with the $\ell_{2}$ metric. A sparse and nonnegative combination of dictionary patches is found to match the subject patch is found using an $\ell_{1}$ solver. These same coefficients are then used to combine patches in another dictionary that is physically matched to the first dictionary. This process is shown in several provided examples to be capable of providing intensity normalization, tissue contrast synthesis, and tissue segmentation.

MIMECS shares strong similarities to nonlocal means methods [45], some of which have been applied in the medical imaging community for noise reduction [42], [84], [85], super-resolution [80], and segmentation [39], [41], [81]. Including our earlier conference publications on this topic [47]-[49], [86], MIMECS is the first approach of this class to be proposed and evaluated for contrast synthesis. The use of nonnegative coefficients in combining patches is a important aspect, heretofore not considered, in using the patch-based framework for contrast synthesis. As well, the proposed dictionary selection technique, which uses a higher-dimensional space to normalize patches, is novel and important in practical scenarios since it avoids prior segmentation (as we used in earlier reports) or atlas selection training. Finally, the importance of sparsity was demonstrated in Section IV-C and Fig. 3.

Comparisons with histogram matching methods (cf. [25], [87]) were omitted from this paper for length considerations, but results are included in [65] and previous conference reports [47]-[49]. Histogram matching methods are used in nearly every neuroimage processing pipeline and remain important. In fact, MIMECS depends on a crude histogram matching method (linear normalization to the WM peak) at its outset. But histogram matching has serious limitations. For example, histogram matching in its most basic form changes the intensities of the subject image so that its histogram is exactly equal to that of the target. If a simple tissue classifier (such as the EM algorithm for a Gaussian mixture model) were applied to these two images, then the computed volumes of the corresponding 
tissue classes would be identical. This process would therefore disguise potential differences in a population of subject images. More sophisticated histogram matching methods have been designed, of course, and their results are more sensible. But the whole approach becomes more suspect as the pulse sequences vary significantly and it is entirely wrong to perform histogram matching between images that have fundamentally different tissue contrasts. MIMECS therefore provides an alternative to histogram matching which does not suffer from these fundamental problems.

Our results demonstrate a collection of potential applications of MIMECS. We particularly emphasize the potential for MIMECS to solve the "missing" or "mis-matched" tissue contrast problem in routine neuroimage processing tasks. For example, the BLSA longitudinal study has been active for so long that the SPGR images that were acquired at $1.5 \mathrm{~T}$ on older scanners has been replaced by MPRAGE images that are acquired at 3.0T on modern scanners. MIMECS offers a straightforward way to carry out consistent volumetric analysis across this large time span. The use of diverse data from multiple institutions and multiple studies creates similar difficulties, as illustrated by our average atlas and segmentation bias examples. These examples showed how MIMECS could be used to synthesize "like" data for potential improvement in the statistical analysis of anatomical shape differences through average atlasing or for merging volumetric analysis of normal subjects for greater power in cross-sectional studies.

The average atlas example demonstrated how registration could be improved by synthesizing images having the same tissue contrast. This was taken a step further with the example involving geometric distortion correction in diffusion MRI. Taken together, these two examples reveal a new approach to multimodal registration, one in which a "proxy" image is synthesized from either the subject or target image and registration is carried out using a simple image similarity criterion such as cross-correlation. This approach deserves further scrutiny since it provides an alternative to the mutual information similarity metric, which is problematic to optimize and often fails to provide accurate results, especially in deformable registration applications.

Super-resolution algorithms, and especially patch-based methods have gained a lot of attention in recent years [81], [83]. The MIMECS framework has strong similarity to other patch-based methods, but offers a twist - direct synthesis of an alternate tissue contrast - that could be advantageous. In fact, it is not difficult to envision putting some of the fundamental notions together, for example, having a multi-resolution atlas within the MIMECS framework. Again, certain elements of the MIMECS framework-nonnegative patch combination and patch normalization in a higher-dimensional space - offer immediate advantages.

Use of patches for direct segmentation has been previously reported [81]. The advantages of MIMECS for this purpose are not clearly established in this paper as we did not include a comparison with these particular methods. However, our comparison to established state-of-the-art segmentation algorithms demonstrates a strong potential for its use in this way in the future. Like other patch-based methods, the use of examples rather than generative models may be advantageous when models are difficult to ascertain with precision as is often the case in medical imaging. A key limitation of this approach at present is that it provides only a tissue classification, not a structural labeling. FreeSurfer, to which it is compared subdivides the GM into anatomical structures with labels, a critically important set in many neuroimaging analyses.

Our final experiment involved FLAIR image synthesis, and it provides a cautionary lesson about the limitations of MIMECS. The FLAIR pulse sequence is unique in its ability to reveal WM lesions by nullifying the CSF signal using a $180^{\circ}$ inversion recovery pulse. This accentuates the lesion signal relative to all other brain intensities. It is a nonlinear relationship to the signal coming from other pulse sequences because of the inversion recovery step, and this is (partly) what makes it impossible to synthesize using MIMECS directly. Lesions are not invisible in MPRAGE and T2-w images, but they are not distinguishable from GM by intensity and local pattern alone. We note that PD images, typically collected along with the T2-w images, do not help us since the lesions in a PD image also look like GM.

While we did illustrate how synthesizing images containing the absence of lesions could potentially be useful in lesion detection, the question still remains as to whether alternate methods or different source images might make FLAIR synthesis possible. It is also natural to ask what other anatomical features or pathologies might not be synthesized using standard MIMECS techniques and how these omissions might affect subsequent image processing steps such as segmentation and registration.

In summary, our case studies demonstrate the immediate utility of MIMECS for a wide variety of neuroimage processing tasks. There remains a great deal of flexibility in its use, particularly in the choice of atlas and source image(s) and new applications can be expected to be found. MIMECS represents a new class of neuroimage processing methods with a potentially rich future.

\section{ACKNOWLEDGMENT}

The authors would like to thank A. Jog for his help in coding the k-d tree and to D. L. Pham for his insightful comments about this manuscript during its preparation. The authors would also like to thank the BLSA participants and neuroimaging staff for their dedication to these studies. Some data used for this study were downloaded from the Biomedical Informatics Research Network (BIRN) Data Repository (http://fbirnbdr.nbirn.net:8080/BDR), supported by grants to the BIRN Coordinating Center (U24-RR019701), Function BIRN (U24-RR021992), Morphometry BIRN (U24-RR021382), and Mouse BIRN (U24-RR021760) Testbeds, funded by the National Center for Research Resources at the National Institutes of Health, U.S.A.

\section{REFERENCES}

[1] J. C. Bezdek, L. O. Hall, and L. P. Clarke, "Review of MR image segmentation techniques using pattern recognition," Med. Physics, vol. 20, no. 4, pp. 1033-1048, 1993 .

[2] P. L. Bazin and D. L. Pham, "Topology-preserving tissue classification of magnetic resonance brain images," IEEE Trans. Med. Imag., vol. 26, no. 4, pp. 487-496, Apr. 2007. 
[3] S. Roy, H. Agarwal, A. Carass, Y. Bai, D. L. Pham, and J. L. Prince, "Fuzzy c-means with variable compactness," in Intl. Sym. on Biomed. Imag. (ISBI), May 2008, pp. 452-455.

[4] K. A. Clark, R. P. Woods, D. A. Rottenberg, A. W. Toga, and J. C. Mazziotta, "Impact of acquisition protocols and processing streams on tissue segmentation of T1 weighted MR images," NeuroImage, vol. 29, no. 1, pp. 185-202, 2006.

[5] K. Boesen, K. Rehm, K. Schaper, S. Stoltzner, R. Woods, E. Lüders, and D. Rottenberg, "Quantitative comparison of four brain extraction algorithms," NeuroImage, vol. 22, no. 3, pp. 1255-1261, July 2004.

[6] S. Roy, A. Carass, P. L. Bazin, S. M. Resnick, and J. L. Prince, "Consistent segmentation using a rician classifier," Medical Image Analysis, vol. 16, no. 2, pp. 524-535, 2012.

[7] B. B. Avants, C. L. Epstein, M. Grossman, and J. C. Gee, "Symmetric diffeomorphic image registration with cross-correlation: Evaluating automated labeling of elderly and neurodegenerative brain," Medical Image Analysis, vol. 12, no. 1, pp. 26-41, 2008.

[8] G. K. Rohde, A. Aldroubi, and B. M. Dawant, "The adaptive bases algorithm for intensity-based nonrigid image registration," IEEE Trans. Med. Imag., vol. 22, pp. 1470-1479, 2003.

[9] C. Rorden, L. Bonilha, J. Fridriksson, B. Bender, and H. Karnath, "Agespecific CT and MRI templates for spatial normalization," NeuroImage, vol. 61, no. 4, pp. 957-966, 2012.

[10] M. Chen, A. Carass, J. Bogovic, and P.-L. B. J. L. Prince, "Distance transforms in multi channel MR image registration," in Proceedings of SPIE Medical Imaging (SPIE-MI 2011), Lake Buena Vista, FL, Feb. 12-17, 2011, 2011.

[11] L. M. Ellingsen and J. L. Prince, "Mjolnir: Extending HAMMER using a diffusion transformation model and histogram equalization for deformable image registration," International Journal of Biomedical Imaging, vol. 2009, no. 281615, 2009.

[12] S. M. Resnick, D. L. Pham, M. A. Kraut, A. B. Zonderman, and C. Davatzikos, "Longitudinal magnetic resonance imaging studies of older adults: A shrinking brain," Journal of Neuroscience, vol. 23, no. 8, pp. 3295-3301, 2003.

[13] M. Thambisetty, J. Wan, A. Carass, Y. An, J. L. Prince, and S. M. Resnick, "Longitudinal changes in cortical thickness associated with normal aging," NeuroImage, vol. 52, no. 4, pp. 1215-1223, 2010.

[14] O. Querbes, F. Aubry, J. Pariente, J. Lotterie, J. Démonet, V. Duret, M. Puel, I. Berry, J.-C. Fort, and P. Celsis, "Early diagnosis of Alzheimer's disease using cortical thickness: Impact of cognitive reserve," Brain, vol. 132, no. 8, pp. 2036-2047, 2009, and The Alzheimer's Disease Neuroimaging Initiative.

[15] N. Raz, K. M. Rodrigue, and J. D. Acker, "Hypertension and the brain: Vulnerability of the prefrontal regions and executive functions," $\mathrm{Be}$ havioral Neuroscience, vol. 117, no. 6, pp. 1169-1180, 2003.

[16] R. A. Heckemann, S. Keihaninejad, P. Aljabar, D. Rueckert, J. V. Hajnal, and A. Hammers, "Improving intersubject image registration using tissue-class information benefits robustness and accuracy of multi-atlas based anatomical segmentation," NeuroImage, vol. 51, no. 1, pp. 221-227, 2010, and The Alzheimer's Disease Neuroimaging Initiative.

[17] P. G. Unschuld, R. A. E. Edden, A. Carass, X. Liu, M. Shanahan, X. Wang, K. Oishi, J. Brandt, S. S. Bassett, G. W. Redgrave, R. L. Margolis, P. C. M. van Zijl, P. B. Barker, and C. A. Ross, "Brain metabolite alterations and cognitive dysfunction in early Huntington's disease,' Movement Disorders, vol. 27, no. 7, pp. 895-902, June 2012.

[18] L. Friedman, H. Stern, G. G. Brown, D. H. Mathalon, J. Turner, G. H. Glover, R. L. Gollub, J. Lauriello, K. O. Lim, T. Cannon, D. N. Greve, H. J. Bockholt, A. Belger, B. Mueller, M. J. Doty, J. He, W. Wells, P. Smyth, S. Pieper, S. Kim, M. Kubicki, M. Vangel, and S. G. Potkin, "Test-retest and between-site reliability in a multicenter fMRI study," Human Brain Mapping, vol. 29, no. 8, pp. 958-972, Aug. 2008.

[19] C. Kawas, S. Gary, R. Brookmeyer, J. Fozard, and A. Zonderman, "Age-specific incidence rates of Alzheimer's disease: The baltimore longitudinal study of aging," Neurology, vol. 54, no. 11, pp. 2072-2077, June 2000.

[20] S. G. Mueller, M. W. Weiner, L. J. Thal, R. C. Petersen, C. Jack, W. Jagust, J. Q. Trojanowski, A. W. Toga, and L. Beckett, "The Alzheimer's disease neuroimaging initiative," Neuroimaging Clin. N. Am., vol. 15, no. 4, pp. 869-877, Nov. 2005

[21] C. Walter, M. Kruessell, A. Gindele, H. G. Brochhagen, A. Gossmann, and P. Landwehr, "Imaging of renal lesions: Evaluation of fast mri and helical ct.," $\mathrm{Br}$. J. Radiol., vol. 76, no. 910, pp. 696-703, Oct. 2003.
[22] J. P. Bergeest and F. Jäger, "A comparison of five methods for signal intensity standardization in MRI," in Bildverarbeitung für die Medizin 2008. : Springer Berlin Heidelberg, 2008, ch. 8, pp. 36-40, ser. Informatik Aktuell.

[23] F. Jäger, L. Nyúl, B. Frericks, F. Wacker, and J. Hornegger, "Whole body MRI intensity standardization," in Bildverarbeitung für die Medizin 2008. : Springer Berlin Heidelberg, 2007, ch. 20, pp. 459-463, ser. Informatik Aktuell.

[24] L. G. Nyúl and J. K. Udupa, "On standardizing the MR image intensity scale," Mag. Reson. Med., vol. 42, no. 6, pp. 1072-1081, 1999.

[25] L. G. Nyúl, J. K. Udupa, and X. Zhang, "New variants of a method of MRI scale standardization," IEEE Trans. Med. Imag., vol. 19, no. 2, pp. 143-150, Feb. 2000.

[26] A. Madabhushi and J. K. Udupa, "New methods of MR image intensity standardization via generalized scale," Med. Physics, vol. 33, no. 9, pp 3426-3434, Sept. 2006.

[27] R. He, S. Datta, G. Tao, and P. A. Narayana, "Information measuresbased intensity standardization of MRI," in Intl. Conf. Engg. in Med. and Biology Soc., Aug. 2008, pp. 2233-2236.

[28] J. D. Christensen, "Normalization of brain magnetic resonance images using histogram even-order derivative analysis," Mag. Reson. Im., vol. 21, no. 7, pp. 817-820, Sept. 2003.

[29] N. L. Weisenfeld and S. K. Warfield, "Normalization of joint imageintensity statistics in MRI using the kullback-leibler divergence," in Intl. Symp. on Biomed. Imag. (ISBI), Apr. 2004, vol. 1, pp. 101-104.

[30] L. M. Ellingsen and J. L. Prince, "Mjolnir: Extending HAMMER using a diffusion transformation model and histogram equalization for deformable image registration," Int. J. Biomed. Imaging, vol. 2009, no. 281615, 2009.

[31] Y. Dai, Y. Wang, L. Wang, G. Wu, F. Shi, and D. Shen, "aBEAT: A toolbox for consistent analysis of longitudinal adult brain MRI," PLoS One, vol. 8, no. 4, p. 60344, 2012, and Alzheimer's Disease Neuroimaging Initiative.

[32] X. Han and B. Fischl, "Atlas renormalization for improved brain MR image segmentation across scanner platforms," IEEE Trans. Med. Imag., vol. 26, no. 4, pp. 479-486, 2007.

[33] B. Fischl, D. H. Salat, A. J. W. van der Kouwe, N. Makris, F. Ségonne, B. T. Quinn, and A. M. Dale, "Sequence-independent segmentation of magnetic resonance images," NeuroImage, vol. 23, no. Suppl. 1, pp. S69-84, 2004

[34] J. L. Prince, Q. Tan, and D. L. Pham, "Optimization of MR pulse sequences for bayesian image segmentation," Med. Physics, vol. 22, no. 10, pp. 1651-1656, Oct. 1995.

[35] R. Deichmann, C. D. Good, O. Josephs, J. Ashburner, and R. Turner, "Optimization of 3-D MP-RAGE sequences for structural brain imaging," Neurolmage, vol. 12, no. 1, pp. 112-127, July 2000.

[36] M. I. Miller, G. E. Christensen, Y. Amit, and U. Grenander, "Mathematical textbook of deformable neuroanatomies," Proc. Natl. Acad. Sci., vol. 90, no. 24, pp. 11944-11948, Dec. 1993.

[37] M. R. Sabuncu, B. T. T. Yeo, K. Van Leemput, B. Fischl, and P. Golland, "A generative model for image segmentation based on label fusion," IEEE Trans. Med. Imag., vol. 29, no. 10, pp. 1714-1729, 2010.

[38] F. Rousseau, "Brain hallucination," in Proc. of the 10th European Conf. on Comp. Vision, 2008, pp. 497-508.

[39] P. Coupé, S. F. Eskildsen, J. V. Manjn, V. S. Fonov, and D. L. Collins, "Simultaneous segmentation and grading of anatomical structures for patient's classification: Application to Alzheimer's disease," NeuroImage, vol. 59, no. 4, pp. 3736-3747, 2012, and the Alzheimer's disease Neuroimaging Initiative.

[40] A. Buades, B. Coll, and J. M. Morel, "A non-local algorithm for image denoising," IEEE Comp. Vision and Patt. Recog., vol. 2, pp. 60-65, 2005.

[41] S. Hu, P. Coupé, J. C. Pruessner, and D. L. Collins, "Nonlocal regularization for active appearance model: Application to medial temporal lobe segmentation," Human Brain Mapping, vol. 00, no. 00, p. $00,2012$.

[42] C. Kervrann, J. Boulanger, and P. Coupé, "Bayesian non-local means filter, image redundancy and adaptive dictionaries for noise removal," in Proc. of 1st International Conference on Scale Space and Variational Methods in Comp. Vision, 2007, vol. 4485, pp. 520-532.

[43] J. Yang, J. Wright, T. S. Huang, and Y. Ma, "Image super-resolution via sparse representation," IEEE Trans. Imag. Proc., vol. 19, no. 11, pp. 2861-2873, Nov. 2010.

[44] E. J. Candes, J. K. Romberg, and T. Tao, "Stable signal recovery from incomplete and inaccurate measurements," Comm. on Pure and Appl. Math., vol. 59, no. 8, pp. 1207-1223, Aug. 2006. 
[45] J. Mairal, F. Bach, J. Ponce, G. Sapiro, and A. Zisserman, "Non-local sparse models for image restoration," in IEEE Intl. Conf. on Comp. Vision, 2009, pp. 2272-2279.

[46] M. Elad and M. Aharon, "Image denoising via sparse and redundant representations over learned dictionaries," IEEE Trans. Imag. Proc., vol. 15, no. 12, pp. 3736-3745, 2006.

[47] S. Roy, A. Carass, and J. L. Prince, "A compressed sensing approach for MR tissue contrast synthesis," in Inf. Proc. in Med. Imaging (IPMI), 2011, pp. 371-383.

[48] S. Roy, A. Carass, and J. L. Prince, "Synthesizing MR contrast and resolution through a patch matching technique," in Proceedings of SPIE Medical Imaging (SPIE), 2010, p. 7623.

[49] S. Roy, A. Carass, and J. L. Prince, "Compressed sensing based intensity non-uniformity correction," in Intl. Sym. on Biomed. Imag. (ISBI), Apr. 2011, pp. 101-104.

[50] J. Mairal, G. Sapiro, and M. Elad, "Learning multiscale sparse representations for image and video restoration," Multiscale Model. Simul., vol. 7, no. 1, pp. 214-241, 2008.

[51] M. Elad and A. Feuer, "Restoration of a single superresolution image from several blurred, noisy, and undersampled measured images," IEEE Trans. Imag. Proc., vol. 6, no. 12, pp. 1646-1658, 1997.

[52] M. Aharon, M. Elad, and A. Bruckstein, "K-SVD: An algorithm for designing overcomplete dictionaries for sparse representation," IEEE Trans. Sig. Proc., vol. 54, no. 11, pp. 4311-4322, 2006.

[53] V. Kwatra, A. Schödl, I. Essa, G. Turk, and A. Bobick, "Graphcut textures: Image and video synthesis using graph cuts," ACM Trans. on Graphics, vol. 22, no. 3, pp. 277-286, 2003.

[54] A. Hertzmann, C. E. Jacobs, N. Oliver, B. Curless, and D. H. Salesin, "Image analogies," in Proc. of 28th Ann. Conf. on Comp. Graphics and Interactive Techniques, ser. SIGGRAPH'01, 2001, pp. 327-340.

[55] D. L. Donoho, "For most large underdetermined systems of linear equations, the minimal $\ell_{1}$-norm near-solution approximates the sparsest near-solution," Communications on Pure and Applied Mathematics, vol. 59, no. 7, pp. 907-934, 2006.

[56] R. Tibshirani, "Regression shrinkage and selection via the lasso," Journal of Royal Stat. Soc. Series B (Methodological), vol. 58, no. 1, pp. 267-288, 1996.

[57] D. L. Donoho and J. Tanner, "Sparse nonnegative solution of underdetermined linear equations by linear programming," Proc. Natl. Acad. Sci., vol. 102, no. 27, pp. 9446-9451, 2005.

[58] K. Koh, S. Kim, and S. Boyd, "An interior-point method for largescale 11-regularized logistic regression," Journal of Machine Learning Research, vol. 8, pp. 1519-1555, 2007.

[59] M. Grant and S. Boyd, "Graph implementations for nonsmooth convex programs," in Recent Advances in Learning and Control. : : SpringerVerlag Limited, 2008, pp. 95-110, ser. Lecture Notes in Control and Information Sciences.

[60] M. Elad, P. Milanfar, and R. Rubinstein, "Analysis versus synthesis in signal priors," Inverse Problems, vol. 23, no. 3, p. 947968, 2007.

[61] M. W. Seeger, "Bayesian inference and optimal design for the sparse linear model," Journal of Machine Learning Research, vol. 9, pp. 759-813, 2008.

[62] J. H. Friedman, J. L. Bentley, and R. A. Finkel, "An algorithm for finding best matches in logarithmic expected time," ACM Trans. on Mathematical Software, vol. 3, no. 3, pp. 209-226, 1977.

[63] C. A. Cocosco, V. Kollokian, R. K. S. Kwan, and A. C. Evans, "BrainWeb: Online interface to a 3-D MRI simulated brain database," Neurolmage, vol. 5, no. 4, p. S425, 1997.

[64] B. A. Landman, A. J. Huang, A. Gifford, D. S. Vikram, I. L. Lim, J. A. D. Farrell, J. A. Bogovic, J. Hua, M. Chen, S. Jarso, S. A. Smith, S. Joel, S. Mori, J. J. Pekar, P. B. Barker, J. L. Prince, and P. C. M. van Zijl, "Multi-parametric neuroimaging reproducibility: A 3-T resource study," NeuroImage, vol. 54, no. 4, pp. 2854-2866, 2011.

[65] S. Roy, "MR Image Contrast Synthesis for Consistent Segmentation" Ph.D. dissertation, Johns Hopkins University, , 2013 [Online]. Available: http://iacl.ece.jhu.edu/ roy/roy_thesis_jhu2013.pdf, [Online]. Available:

[66] A. Carass, M. B. Wheeler, J. Cuzzocreo, P.-L. Bazin, S. S. Bassett, and J. L. Prince, "A joint registration and segmentation approach to skull stripping," in Intl. Sym. on Biomed. Imag. (ISBI), Apr. 2007, pp. $656-659$.

[67] A. Carass, J. Cuzzocre, M. B. Wheeler, P. L. Bazin, S. M. Resnick, and J. L. Prince, "Simple paradigm for extra-cerebral tissue removal: Algorithm and analysis," NeuroImage, vol. 56, no. 4, pp. 1982-1992, 2011.
[68] J. G. Sled, A. P. Zijdenbos, and A. C. Evans, "A non-parametric method for automatic correction of intensity non-uniformity in MRI data," IEEE Trans. Med. Imag., vol. 17, no. 1, pp. 87-97, 1998.

[69] A. Moghekar, M. Kraut, W. Elkins, J. Troncoso, A. B. Zonderman, S. M. Resnick, and R. J. O'Brien, "Cerebral white matter disease is associated with Alzheimer pathology in a prospective cohort," Alzheimer's \& Dementia, vol. 8, no. 5, pp. S71-S77, 2012.

[70] Z. Xue, D. Shen, and C. Davatzikos, "CLASSIC: Consistent Longitudinal Alignment and Segmentation for Serial Image Computing," NeuroImage, vol. 30, no. 2, pp. 388-399, 2006.

[71] M. Reuter, N. J. Schmansky, H. D. Rosas, and B. Fischl, "Withinsubject template estimation for unbiased longitudinal image analysis," NeuroImage, vol. 61, no. 4, pp. 1402-1418, 2012.

[72] S. Roy, A. Carass, and J. L. Prince, "Longitudinal intensity normalization of magnetic resonance images using patches," in Proceedings of SPIE Medical Imaging (SPIE-MI 2013), Lake Buena Vista, FL, Feb. 9-14, 2013, p. 86691J, 2013.

[73] D. S. Marcus, T. H. Wang, J. Parker, J. G. Csernansky, J. C. Morris, and R. L. Buckner, "Open Access Series of Imaging Studies (OASIS): Crosssectional MRI data in young, middle aged, nondemented, and demented older adult," Journal of Cognitive Neuroscience, vol. 19, no. 9, pp. 1498-1507, Sept. 2007.

[74] A. M. Dale, B. Fischl, and M. I. Sereno, "Cortical surface-based analysis I: Segmentation and surface reconstruction," NeuroImage, vol. 9, no. 2, pp. 179-194, 1999.

[75] F. Klauschen, A. Goldman, V. Barra, A. Meyer-Lindenberg, and A. Lundervold, "Evaluation of automated brain MR image segmentation and volumetry methods," Human Brain Mapping, vol. 30, no. 4, pp. $1310-1327,2009$

[76] P. Jezzard and R. S. Balaban, "Correction for geometric distortion in echo planar images from $B_{0}$ field variations," Mag. Reson. Med., vol. 34, no. 1, pp. 65-73, 1995.

[77] S. Ardekani and U. Sinha, "Geometric distortion correction of highresolution 3T diffusion tensor brain images," Mag. Reson. Med., vol. 54, no. 5, pp. 1163-1171, 2005.

[78] J. Souplet, C. Lebrun, N. Ayache, and G. Malandain, "An automatic segmentation of T2-FLAIR multiple sclerosis lesions," in Multiple Sclerosis Lesion Segmentation Challenge Workshop (MICCAI 2008 Workshop), New York, Sep. 2008, pp. 1-11.

[79] Y. Wu, S. K. Warfield, I. L. Tan, W. M. Wells, D. S. Meier, R. A. van Schijndel, F. Barkhof, and C. R. G. Guttmann, "Automated segmentation of multiple sclerosis lesion subtypes with multichannel MRI," NeuroImage, vol. 32, no. 3, pp. 1205-1215, 2006.

[80] J. V. Manjon, P. Coupé, A. Buades, V. Fonovan, D. L. Collins, and M. Robles, "Non-local MRI upsampling," Med. Image Anal., vol. 14, no. 2, pp. 784-792, 2010.

[81] P. Coupé, J. V. Manjon, J. Pruessner, M. Robles, V. Fonov, and D. L. Collins, "Patch-based segmentation using expert priors: Application to hippocampus and ventricle segmentation," NeuroImage, vol. 54, no. 2, pp. 940-954, 2011.

[82] S. F. Eskildsen, P. Coupé, V. Fonov, J. V. Manjon, K. K. Leung, N. Guizard, S. N. Wassef, L. R. Ostergaard, and D. L. Collins, Alzheimer's Disease Neuroimaging Initiative, "BEaST: Brain extraction based on nonlocal segmentation technique," NeuroImage, vol. 59, no. 3, pp. 2362-2373, 2012 .

[83] F. Rousseau, P. A. Habas, and C. Studholme, "A supervised patchbased approach for human brain labeling," IEEE Trans. Med. Imag., vol. 30, no. 10, pp. 1852-1862, Oct. 2011.

[84] J. V. Manjón, J. Carbonell-Caballero, J. J. Lull, G. Garcia-Marti, L. Marti-Bonmati, and M. Robles, "MRI denoising using non-local means," Med. Image Anal., vol. 12, no. 4, pp. 514-523, 2008.

[85] N. Wiest-Daessle, S. Prima, P. Coupé, S. P. Morrissey, and C. Barillot, "Rician noise removal by non-local means filtering for low signaltonoise ratio MRI: Applications to DT-MRI," in Med. Image Comp. Comp. Asst. Intervent., 2008, vol. 11, pp. 171-179.

[86] S. Roy, A. Carass, N. Shiee, D. L. Pham, and J. L. Prince, "MR contrast synthesis for lesion segmentation," in Proc. Int. Symp. Biomed. Imag., 2010, pp. 932-935.

[87] L. Wang, H. Lai, G. J. Barker, D. H. Miller, and P. S. Tofts, "Correction for variations in MRI scanner sensitivity in brain studies with histogram matching," Mag. Reson. Med., vol. 39, no. 2, pp. 322-327, Feb. 1998. 